This is the peer reviewed version of: Khamis, M. M., Adamko, D. J. and El-Aneed, A. (2015), Mass Spec Rev., published in final form at https://doi.org/10.1002/mas.21455.

This article may be used for non-commercial purposes in accordance with Wiley Terms and Conditions for SelfArchiving.

\title{
Mass Spectrometric Based Approaches in Urine Metabolomics and Biomarker Discovery
}

Mona M. Khamis ${ }^{1,2}$, Darryl J. Adamko ${ }^{3}$ and Anas El-Aneed ${ }^{1 *}$

${ }^{1}$ College of Pharmacy and Nutrition, University of Saskatchewan, 107 Wiggins Rd, Saskatoon, SK, S7N 5E5 Canada

${ }^{2}$ Faculty of Pharmacy, Alexandria University, Alexandria 21521, Egypt

${ }^{3}$ Department of Pediatrics, College of Medicine, University of Saskatchewan, 103 Hospital Drive, Saskatoon, SK, Canada

To whom correspondence should be addressed:

Anas El-Aneed, Associate Professor

College of Pharmacy and Nutrition

University of Saskatchewan

107 Wiggins Rd, Saskatoon, SK, S7N 5E5 Canada

Phone: 306-966-2013

Fax: 306-966-6377

e-mail: anas.el-aneed@usask.ca

\begin{abstract}
Urine metabolomics has recently emerged as a prominent field for the discovery of non-invasive biomarkers that can detect subtle metabolic discrepancies in response to a specific disease or therapeutic intervention. Urine, compared to other biofluids, is characterized by its ease of collection, richness in metabolites and its ability to reflect imbalances of all biochemical pathways within the body. Following urine collection for metabolomic analysis, samples must be immediately frozen to quench any biogenic and/or non-biogenic chemical reactions. According to the aim of the experiment; sample preparation can vary from simple procedures such as filtration to more specific extraction protocols such as liquid-liquid extraction. Due to the lack of comprehensive studies on urine metabolome stability, higher storage temperatures (i.e. $4{ }^{\circ} \mathrm{C}$ ) and repetitive freeze-thaw cycles should be avoided. To date, among all analytical techniques, mass spectrometry (MS) provides the best sensitivity, selectivity and identification capabilities to analyze the majority of the metabolite composition in the urine. Combined with the qualitative and quantitative capabilities of MS, and due to the continuous improvements in its
\end{abstract}


This is the peer reviewed version of: Khamis, M. M., Adamko, D. J. and El-Aneed, A. (2015), Mass Spec Rev., published in final form at https://doi.org/10.1002/mas.21455.

related technologies (i.e. ultra high-performance liquid chromatography [UPLC] and hydrophilic interaction liquid chromatography [HILIC]), liquid chromatography (LC)-MS is unequivocally the most utilized and the most informative analytical tool employed in urine metabolomics. Furthermore, differential isotope tagging techniques has provided a solution to ion suppression from urine matrix thus allowing for quantitative analysis. In addition to LC-MS, other MS-based technologies have been utilized in urine metabolomics. These include direct injection (infusion)-MS, capillary electrophoresisMS and gas chromatography-MS. In this article, the current progresses of different MS-based techniques in exploring the urine metabolome as well as the recent findings in providing potentially diagnostic urinary biomarkers are discussed.

Keywords: Metabolome, Urine, Untargeted, Targeted, Biomarker, Mass Spectrometry

\section{INTRODUCTION}

The discovery, validation and quantification of new biomarkers or "biological markers" are essential cornerstones in the field of biomedical research. One such target for biomarker identification is metabolites. Metabolomics is the study of metabolic profiles in a biological system and it is the endpoint in the omics cascade that starts with activated genes (genome) along with gene transcripts (transcriptome) and proteins (proteome) (Dettmer, Aronov \& Hammock, 2007; Dunn et al., 2011; Jain, 2010; Roberts et al., 2012). Changes at the metabolite level are natural outcomes of the onset and prognosis of many diseases and such changes often appear in biological fluids before the appearance of clinical symptoms (Van, Veenstra \& Issaq, 2011). For this reason, the discovery of new diagnostic and prognostic biomarkers whether based on genomics (Borovecki et al., 2005; Hu et al., 2007; Moore et al., 2008), proteomics (Hu et al., 2007; Zhang et al., 2004) or metabolomics (Gowda et al., 2008; Zhang et al., 2012b) can enhance the understanding of human diseases and eventually improve the quality of life of patients.

Currently, there are two main approaches by which metabolomic studies can be conducted, namely metabolic profiling and fingerprinting (Fig 1) (Dettmer, Aronov \& Hammock, 2007). Metabolic profiling or the "targeted approach" aims at identifying and quantifying a preselected category of metabolites within a sample (Dettmer, Aronov \& Hammock, 2007; Griffiths et al., 2010; Patti, Yanes \& Siuzdak, 2012; Roberts et al., 2012; Theodoridis, Gika \& Wilson, 2011). Selection criteria can be based on either a common chemical functional group such as amino or carboxylic acid functionalities, or specific biochemical pathways. This targeted analysis is usually driven by a proposed hypotheses or a biochemical question (Fig 1) (Dettmer, Aronov \& Hammock, 2007; Dunn et al., 2011; Griffiths et al., 2010; Patti, Yanes \& Siuzdak, 2012; Theodoridis, Gika \& Wilson, 2011). On the other hand, fingerprinting or the "untargeted or global approach" compares unquantified metabolic profiles between analogous samples in an attempt of tracking differences in response to some internal or external factors (e.g. a disease, toxin or environmental stimulus) (Dettmer, Aronov \& Hammock, 2007). Metabolic profiling and fingerprinting are complementary and their integrative implementation in biomarker discovery reveals the true power of the metabolome in understanding complex biochemical

pathways. In fact, as seen in Fig 1, biomarker discovery involves sequential steps of first determining differences in the metabolic profiles between samples and then selecting the key altered metabolites as potential biomarkers. Secondly, targeted metabolites are quantified and their differential expression is justified through the understanding of the underlying biochemical pathways. For instance, statins are the first line of treatment of cholesterol induced atherosclerotic-cardiovascular diseases and they are known to elevate hepatic enzymes (Kumar at al., 2010). Therefore, it is necessary to identify biomarkers that 
This is the peer reviewed version of: Khamis, M. M., Adamko, D. J. and El-Aneed, A. (2015), Mass Spec Rev., published in final form at https://doi.org/10.1002/mas.21455.

can indicate the first appearance of adverse effects (i.e. safety biomarkers). In order to detect liver toxicity markers for atorvastatin, hyperlipidemic rats were treated with either $70 \mathrm{mg} / \mathrm{kg}$ or $250 \mathrm{mg} / \mathrm{kg}$ atorvastatin for 7 days. Urinary profiles of these two groups were statistically different than those of control and hyperlipidemic control groups. Subsequently, urinary amino acids and steroidal metabolites, which were altered by the drug induced-liver damage, were quantified in a targeted approach. Six metabolites were identified as potential safety biomarkers for atorvastatin-induced liver toxicity. With the exception of two metabolites, the connections between candidate biomarkers and liver damage were explained (Kumar et al., 2010).

Among the various bodily fluids, urine is an attractive option for metabolome biomarker discovery. It can be readily obtained in large quantities non-invasively with expected high participant compliance. In addition, it poses low risk of infection to researchers (Miller et al., 2004). Assuming normal renal function, the fluid is filtered leaving low cell contamination. Concentrations of metabolites in the urine are often higher than that of plasma, thus providing a richer matrix for analysis (Adamko, et al., 2007; Miller et al., 2004). The use of urine as a source for biomarker discovery will be the focus of this review.

Metabolites contain diverse classes of low-molecular weight compounds ( $<1500 \mathrm{Da})$ such as; amino acids, lipids, organic acids and nucleotides (Dettmer, Aronov \& Hammock, 2007; Wishart et al., 2013). They are of heterogeneous physicochemical properties, and they exist in very wide concentration ranges (pmol-mmol) ( Dettmer, Aronov \& Hammock, 2007; Dunn et al., 2011; Dunn \& Ellis, 2005; Wishart et al., 2013). The Human Metabolome Database (HMDB) is being continuously updated to include all known metabolites; their biofunction, pathways, locations and normal and abnormal concentrations. They are chemically categorized into a hierarchical pattern of kingdoms, super classes, classes and sub classes (Wishart et al., 2013). Unfortunately, unlike genes or proteins, metabolites are not specific to a single metabolicpathway. In most cases, several biochemical reactions can contribute to the production of a single metabolite within the urine (Kumar et al., 2012). Thus, metabolomics has the advantage of seeing the cumulative effect of an altered state within the body either due to drugs, diseases or any other pathophysiological condition. For example, changes in the urinary metabolomic profile have been identified throughout the different stages of pregnancy (Trivedi \& Iles, 2014b). Four metabolites drastically decreased at the end of first trimester, while, an increased expression of a number of metabolites was observed in the mid second trimester. Such study can serve as a platform for the indication of a healthy fetal-maternal metabolism to which other pathologies of pregnancy can be compared to (Trivedi \& Iles, 2014b). On the other hand, in addition to the complex nature of metabolites within urine; genetic and environmental stimuli are also known to influence the normal metabolome profile (Johnson \& Gonzalez, 2012). For instance, based on a 50-metabolite profiling strategy using urine, 60 participants were successfully differentiated based on their age and gender (Slupsky et al., 2007). Another non-targeted metabolome study was able to identify some major alterations in the urine metabolic profiles following whole grain rye and wheat bread consumption (Bondia-Pons et al., 2013).

Based on current technologies and the diversity of the physicochemical properties of metabolites, a single separation or detection technique, which can simultaneously identify all metabolites within a urine sample, is not available (Dettmer, Aronov, Hammock, 2007; Scalbert et al., 2009). Therefore, different analytical approaches must be adopted according to the properties of metabolites being investigated and the specific goal of the experiment ( Dettmer, Aronov, Hammock, 2007; Lei, Huhman, \& Sumner, 2011; Scalbert et al., 2009). Recent improvements in mass spectrometry (MS) have expanded the range of metabolites that can be analyzed in urine for either metabolite identification or quantification. Indeed, MS-based techniques are currently the most prevalent tools employed in 
This is the peer reviewed version of: Khamis, M. M., Adamko, D. J. and El-Aneed, A. (2015), Mass Spec Rev., published in final form at https://doi.org/10.1002/mas.21455.

metabolomics studies (Dunn, 2008; Dunn et al., 2011; Griffiths et al., 2010; Johnson \& Gonzalez, 2012). In this paper, we explore the advancements in MS-based techniques in targeted and untargeted urine metabolomic analysis, while providing comparisons between currently available technologies. In addition, urine handling for biomarker analysis will be reviewed and special emphasis will be given to the current status and the success of urine as a matrix for biomarker discovery.

\section{HANDLING OF URINE SAMPLES FOR MS ANALYSIS}

Bacterial, biogenic enzymatic reactions and non-biogenic oxidation reactions of metabolites are reported to alter the metabolic profile of urine (Bernini et al., 2011; Boomsma et al., 1993; Boyland, Wallace \& Williams, 1955; Fernández-Peralbo \& Luque de Castro, 2012; Ito et al., 2002; Jung \& Pergande, 1983; Sykes, 2007; Udert et al., 2003; Zeisel, DaCosta \& Fox, 1985). Bacterial enzymes are known to be active towards several metabolites such as urea (Udert et al., 2003), choline (Zeisel, DaCosta \& Fox, 1985) and creatinine (Ito et al., 2002). Biogenic enzymes, namely glucuronidase and sulphatase maintain their conjugate-hydrolytic activity in normal urine for a period up to 10 days after collection ( Boyland, Wallace \& Williams, 1955). In addition to enzymatic degradations, some labile compounds like catecholamines are easily oxidized into their corresponding quinones when kept at 37 ${ }^{\circ} \mathrm{C}$ for $1-2$ days or at $20{ }^{\circ} \mathrm{C}$ for 4-8 days (Boomsma et al., 1993). Therefore, the main target of urine sample collection and preparation is to ideally maintain the metabolomic state of the original sample, and to halt chemical/enzymatic reactions in urine that might give a misleading metabolomic picture of analyzed samples (Boomsma et al., 1993; Dunn et al., 2011; Sykes, 2007; Vuckovic, 2012). The following sections describe the available sampling modes, proper sample clean-up procedures as well as appropriate conditions for storing urine samples until the time of analysis.

\section{A. Urine Sampling Time}

Urine samples can be collected randomly (Adamko et al., 2007; Saude et al., 2009; Saude et al., 2011; Wittke et al., 2003), at pre-set time intervals (Tyburski et al., 2009; Want et al., 2010), for up to 24 hrs (Holmes et al., 2008; Tyburski et al., 2009; Urpi-Sarda et al., 2009; Want et al., 2010) or for longer periods (e.g. pharmacokinetic studies) (Tyburski et al., 2009). Mid-stream random sampling can be done anytime of the day, and it does not correct for the diurnal variation of excretion of some metabolites (Fernández-Peralbo \& Luque de Castro, 2012). On the contrary, timed sampling is used to monitor the exact excretion pattern of metabolites exhibiting a circadian rhythm. It could also monitor metabolites produced following special diet, xenobiotic consumption, or response to stimulation (e.g. injury) (Fernández-Peralbo \& Luque de Castro, 2012). One drawback of the 24-hrs sample collection technique is that it cannot provide information regarding the diurnal variation of excretion or the timing of excretion in response to stimuli (Fernández-Peralbo \& Luque de Castro, 2012). In contrast, despite being labor intensive, this sampling procedure eliminates the vast variation in urine profile observed at shorter times of collection. As such, it gives the most accurate quantification of clinically important metabolites (Pinto-Sietsma et al., 2000; Remer et al., 2005).

Ultimately, the purpose of the experiment dictates the proper sampling mode. For instance, changes in the urine profile of mice following the exposure to different doses of sub lethal gammaradiation were evaluated. Sampling protocol involved the collection of urine at 4 hrs intervals after exposure for a period of 20 hrs. In addition, subsequent full 24 hrs urine samples were collected at 3, 5, 7 and 9 days following exposure (Tyburski et al., 2009). Six purine and pyrimidine derivatives exhibited significantly altered excretion behavior and were suggested as possible biomarkers for sub lethal radiation exposure. Elevated metabolites exhibited a time dependent excretion rate, where peak concentrations were detected in urine at 8-12 hrs after exposure and baseline levels were restored by 36 
This is the peer reviewed version of: Khamis, M. M., Adamko, D. J. and El-Aneed, A. (2015), Mass Spec Rev., published in final form at https://doi.org/10.1002/mas.21455.

hrs following irradiation. On the other hand, in the work of Saude et al. (Saude et al., 2009; Saude et al., 2011), urine samples were intentionally collected randomly without any dietary restriction. Statistical analysis of the obtained data revealed sufficiently altered urinary metabolic profiles that allowed the differentiation between healthy and asthma guinea pigs (Saude et al., 2009) and children (Saude et al., 2011) with accuracy of more than $90 \%$.

\section{B. Sample Preparation}

Normal urine is composed of 95\% water, organic compounds, electrolytes and metabolites with lower protein content in comparison to more complex biological fluids such as serum or plasma (Fernández-Peralbo \& Luque de Castro, 2012; Want et al., 2010). Therefore, in general, urine sample preparation is one of the simplest processes employed in bioanalysis and the complexity of this step usually differs according to the aim of the experiment (Dunn et al., 2011; Fernández-Peralbo \& Luque de Castro, 2012). Sample preparation in non-targeted analysis aims at keeping the sample as intact as possible containing all unknown metabolites with various physicochemical properties (Dunn et al., 2011; Fernández-Peralbo \& Luque de Castro, 2012). The method employed should be non-selective, fast, simple and reproducible (Fernández-Peralbo \& Luque de Castro, 2012). For these reasons, the simplest unselective sample-preparation procedure is sample dilution or ultrafiltration (Vuckovic, 2012). "Dilute and Shoot" is a strategy for the preparation of simple biological matrices as urine. In this approach, the samples are only diluted with the appropriate solvents prior to their injection (shooting) into the analytical system(Martínez-Lozano \& Rus, 2010; Vuckovic, 2012). On the other hand, ultrafiltration uses molecular weight cut-off filters of $3000 \mathrm{Da}$ or higher providing the best coverage for low-molecular weight compounds in global urine metabolome analysis (Pesek et al., 2009; Vuckovic, 2012).

In targeted analysis, however, where a limited group of metabolites are investigated, selective analytical extraction techniques, such as solid phase extraction (SPE) or liquid-liquid extraction (LLE) can be used for pre-concentration and clean-up purposes (Dunn et al., 2011; Fernández-Peralbo \& Luque de Castro, 2012; Peng \& Li, 2013; Urpi-Sarda et al., 2009). Phenolic conjugated metabolites and colonic microbial metabolites that are produced due to regular cocoa consumption were extracted from plasma and urine using simple and fast SPE procedures (Urpi-Sarda et al., 2009). Quantitative analysis of the extracted urine samples using liquid chromatography-tandem mass spectrometry (LC-MS/MS) revealed the significant increase of phase II metabolites including glucuronide and sulfate conjugates of epicatechin, $O$-methyl-epicatechin, 5-(3',4'-dihydroxyphenyl)- $\gamma$-valerolactone and 5-(3'-methoxy-4'hydroxyphenyl)- $\gamma$-valerolactone. The findings suggest the aptness of these compounds as urinary biomarkers of the consumption of flavanol rich food (Urpi-Sarda et al., 2009).

Despite being an older technique relative to SPE, LLE is still capable of providing clean samples for metabolome analysis. For example, carboxylic acids urinary metabolic profiles generated using precolumn derivatization reaction followed by reverse-phase liquid chromatography (RPLC)-Fourier transform ion cyclotron resonance mass spectrometry (FTICR-MS) were compared with and without the incorporation of a LLE clean-up step (Fig 2) (Peng \& Li, 2013). It was shown that the additional preconcentration improved the sensitivity of quantification of organic acid metabolites. Moreover, water in urine, which reacts with the derivatizing reagent and thus reduces the reaction efficiency, was eliminated through the additional LLE process. Interference from highly abundant amino acids was also removed allowing the detection of other acids present in low concentrations. As a result, 2491 MS peaks were detected in urine samples after LLE in comparison to 874 peaks detected without extraction (Peng \& Li, 2013). 
This is the peer reviewed version of: Khamis, M. M., Adamko, D. J. and El-Aneed, A. (2015), Mass Spec Rev., published in final form at https://doi.org/10.1002/mas.21455.

Fresh urine is characterized by the presence of human cells, bacteria and non-cellular components including urates and mucus filaments (Bernini et al., 2011; Sykes, 2007). Accordingly, prior to analysis, urine samples can be treated either through filtration, centrifugation or via the addition of a preservative (Sykes, 2007). It was shown that spinning of urine samples at 10,000 rpm for 10 min was less effective in conserving the metabolome composition in comparison to filtration through $0.22 \mu \mathrm{m}$ syringe filter (Sykes, 2007). The addition of sodium azide, a preservative, in a concentration of $10 \mathrm{mM}$ provided better metabolic stability after a 4-week storage period at room temperature in comparison to lower concentrations (Sykes, 2007). Among all preparation methods, filtration showed superior ability to preserve the metabolic profile during storage which can be attributed to bacterial removal. However, the loss of larger metabolites through filtration should be taken into account (Sykes, 2007).

\section{Sample Storage}

Following sample collection, immediate freezing is usually recommended in order to quench any rapid degradation activity such as oxidation of labile metabolites as well as various enzymatic reactions (Bernini et al., 2011; Fernández-Peralbo \& Luque de Castro, 2012; Ryan et al., 2011; Sykes, 2007; van de Merbel, 2008). Sykes et al (Sykes, 2007) demonstrated significant changes in a considerable number of metabolites in both male and female urine samples after a 4-week storage period at $22{ }^{\circ} \mathrm{C}$ using NMR combined with analysis of variance (ANOVA) and t-test statistical tools. Creatinine decreased significantly due to bacterial creatininase activity with the concomitant rise in creatine levels (Sykes, 2007). This instability is concerning as normalization to creatinine, as discussed below, is the most widely employed method for the correction of urine volume variation (Ryan et al., 2011). Storing urine samples at $4{ }^{\circ} \mathrm{C}$ for 4 weeks in the same study resulted in altered urine metabolic profile. However, that variation was less significant in comparison to samples stored at room temperature (Sykes, 2007). Similarly, metabolic changes were observed in urine following a week of storage at $4{ }^{\circ} \mathrm{C}$ using NMR (Lauridsen et al., 2007). A decrease in hippurate concentration was observed with the simultaneous rise in its degradation products; benzoic acid and glycine. Long term sample storage at same conditions (above 1 week) caused further increase in acetate and decrease in histidine and citrate concentrations (Lauridsen et al., 2007). Likewise, another study confirmed the degradation of metabolites and the production of artifacts in urine samples stored at $4{ }^{\circ} \mathrm{C}$ (Trivedi et al., 2012).

On the contrary to the above discussed studies, it was demonstrated that storing samples at $4{ }^{\circ} \mathrm{C}$ for $24 \mathrm{hrs}$ after collection and prior to storage at $-80{ }^{\circ} \mathrm{C}$ did not alter the metabolic profile compared to samples directly stored at $-80{ }^{\circ} \mathrm{C}$ (Dunn et al., 2008). Samples were analyzed using gas chromatography-Time of Flight (GC-ToF) MS and statistical analysis of the results was achieved using univariate statistical analysis as well as principle component analysis (PCA) (Dunn et al., 2008). Interestingly, another study supported the notion of fridge stability by indicating that urine sample storage at $0-4{ }^{\circ} \mathrm{C}$ in a fridge or cooled autosampler for up to 48 hrs maintained the metabolic integrity of the samples (Gika, Theodoridis \& Wilson, 2008b). Analysis was performed using both LC-quadrupole linear ion trap (LTQ) and ultra highperformance liquid chromatography (UPLC)-Quadrupole-Time of Flight (QToF) MS and results were analyzed using PCA (Gika, Theodoridis \& Wilson, 2008b).

Concerning the optimum freezing temperature for long term storage, Gika et al (Gika, Theodoridis \& Wilson, 2008b) proved that the PCA score plots of urine samples stored at either -20 or $80{ }^{\circ} \mathrm{C}$ for a period of up to 6 months did not show any significant differences. This study, however, did not confirm whether or not the stored samples are identical to the original ones at the time of collection ( (Gika, Theodoridis \& Wilson, 2008b). Likewise, Lauridsen and co-workers (Lauridsen et al., 2007) confirmed previous stability results by obtaining identical NMR patterns for urine samples stored for 26 
This is the peer reviewed version of: Khamis, M. M., Adamko, D. J. and El-Aneed, A. (2015), Mass Spec Rev., published in final form at https://doi.org/10.1002/mas.21455.

weeks at either $-25{ }^{\circ} \mathrm{C}$ or $-80^{\circ} \mathrm{C}$. In addition, it was demonstrated that no benefits can be obtained by the addition of $15 \mathrm{mM}$ sodium azide to samples intended to be stored at either temperatures for 26 weeks (Lauridsen et al., 2007). Another study confirmed the necessity of storing urine at $-20{ }^{\circ} \mathrm{C}$ or at even lower temperatures in order to maintain the integrity of the samples (Trivedi et al., 2012). Clearly, stability of metabolites is maintained at lower temperatures of storage; however, a conclusive agreement on urine metabolome behavior at higher storage temperatures $\left(0-4{ }^{\circ} \mathrm{C}\right)$ is lacking. The exact reason beyond these conflicting reports cannot be easily identified. Such variations can be attributed to many variables such as number of participants, time of urine collection, methods of sample preparation and methods of analysis.

\section{Freeze-Thaw stability}

It is recommended that freeze-thaw cycles are to be avoided as much as possible to prevent potential degradation; urine samples should be frozen in a pre-aliquot fashion following collection (Vuckovic, 2012; Want et al., 2010). Urine samples which were frozen at $-80^{\circ} \mathrm{C}$ and thawed twice a week for 4 weeks showed reduced metabolic stability in comparison to non-thawed ones stored at the same temperature (Sykes, 2007). In contrast, Gika et al. demonstrated urine sample stability for up to 9 freezethaw cycles (Gika, Theodoridis \& Wilson, 2008b). It should be noted, however, that samples in the latter study were stored at $-20{ }^{\circ} \mathrm{C}$ and were allowed to thaw at room temperature for $3 \mathrm{~h}$ for 9 days (1 cycle/day) (Gika, Theodoridis \& Wilson, 2008b). The shorter study period (9 days versus 4 weeks) may explain the relative stability of urine samples despite the same number of freeze-thaw cycles. Interestingly, Trivedi et al. evaluated the stability of urine after 5 freeze-thaw cycles from $-20^{\circ} \mathrm{C}$ over 5 days and found that the urinary profiles can be maintained only up to 3 cycles (Trivedi et al., 2012). Again, due to the lack of comprehensive studies on freeze and thaw stability, this procedure should be avoided, or minimized as much as possible in urine metabolome analysis.

\section{MASS SPECTROMETRIC TECHNIQUES EMPLOYED IN URINE METABOLOMIC ANALYSIS}

Compared to other analytical techniques, MS has the advantages of high sensitivity, selectivity and chemical identification capabilities for unknown metabolites (Dunn, 2008; Dunn et al., 2011; Dunn \& Ellis, 2005; Lei, Huhman \& Sumner, 2011). When combined with chromatographic separation, it allows for the detection of thousands of compounds in a global manner as well as the quantification of metabolites in targeted analysis (Dunn et al., 2011; Dunn \& Ellis, 2005). However, quantification necessitates the inclusion of internal standard (IS) to compensate for the variation of response factor due to sample matrix effects (Dunn et al., 2011). A wide variety of ionization sources are available, though electron impact (EI) and electrospray ionization (ESI) are the most commonly used ionization techniques in metabolomic analysis (Dunn et al., 2011). EI is the ionization of choice when GC is utilized, while ESI allows coupling of LC or capillary electrophoresis (CE) to MS (Dunn et al., 2011; Lei, Huhman \& Sumner, 2011). It is often required in the global approach to analyze the same sample in the positive and negative ion modes; such a protocol provides broader metabolome coverage through the detection of two metabolite sets that can substantially differ in chemical structures (Dettmer, Aronov \& Hammock, 2007; Theodoridis, Gika \& Wilson, 2011). ToF, QToF, FTICR-MS and orbitrap are high resolution mass analyzers that allow for the identification of co-eluting metabolites with the same nominal mass. Therefore, these are the analyzers of choice in untargeted metabolomic analysis (Guo et al., 2012; Mikami, Aoki \& Kimura, 2012; Want et al., 2010). Triple quadrupoles (QqQ), however, are more suited for quantitative purposes in targeted analysis (Mikami, Aoki \& Kimura, 2012; Want et al., 2010). Although the use of neutral loss and precursor/product ion scans in urine metabolome analysis is 
This is the peer reviewed version of: Khamis, M. M., Adamko, D. J. and El-Aneed, A. (2015), Mass Spec Rev., published in final form at https://doi.org/10.1002/mas.21455.

reported (Bicker et al. 2005; Lafaye et al., 2004; Wagner et al., 2006), multiple reaction monitoring (MRM) provides the highest sensitivity and a wider dynamic range (Lu, Bennett \& Rabinowitz, 2008).

\section{A. Direct Injection (Infusion) Mass Spectrometry (DIMS)}

In this approach, the diluted urine sample is directly infused into ESI-equipped mass spectrometers without any prior chromatographic separation. Undiluted urine, however, is rarely used due to the high concentration of contained salts which are not normally removed via filtration. Injection can be done via a syringe or in an automated direct flow injection mode (DFI). In DFI, the sample passes through lines of the LC pumping systems without any chromatographic separation thus allowing for the fast and accurate introduction of various solutions from different LC vials placed in the autosampler (Dettmer, Aronov \& Hammock, 2007; Dunn et al., 2008; Dunn et al., 2011). DIMS is a powerful tool for structural elucidation of hundreds of metabolites within a single run. Therefore, with its short analysis time and comparable prediction capabilities to LC-MS, it can be regarded as a "fit for purpose” approach in high throughput untargeted metabolomics (Kirwan et al., 2013). However, despite its high detection power for all contained metabolites, this technique is limited by its inability to distinguish between isobaric molecules such as; stereoisomers (hexose sugars) or structural isomers (leucine and isoleucine), which possess the exact molecular weights (Dettmer, Aronov \& Hammock, 2007; Dunn et al., 2011; Dunn \& Ellis, 2005). In addition, DIMS exhibits remarkably high ionization suppression (Dettmer, Aronov \& Hammock, 2007; Dunn et al., 2011; Dunn \& Ellis, 2005; Want et al., 2010). A 60\% ion suppression of internal standards spiked in rat urine was observed in comparison to standards spiked in saline solution despite the removal of salts from urine using online SPE extraction (Fig 3) (Dettmer, Aronov \& Hammock, 2007). DIMS also can rapidly cause a deterioration of the performance of the instrument due to non-volatile residues (Dettmer, Aronov \& Hammock, 2007). Due to such hurdles, its use is limited to rapid high-throughput screening purposes when a large number of samples are investigated for fingerprinting or putative identification (Mikami, Aoki \& Kimura, 2012; Zhang et al., 2012a). Due to the anticipated increase in metabolomics studies, recent reports investigate possible means to overcome the technical challenges facing the widespread use of DIMS. For instance, non-volatile residues are required to be regularly removed through routinely scheduled maintenance of the ion source (Kirwan et al., 2013). In addition, Kirwan et al. have recently developed computational workflows that significantly decreased the analytical variation to the acceptable levels as per the Food and Drug Administration (FDA) guidelines for new biomarker discovery (Kirwan et al., 2013; Kirwan, 2014).

As in the case of other biological fluids, due to urine complexity, the use of DIMS usually involves the use of high resolution mass analyzers such as FTICR-MS or ToF (Dettmer, Aronov \& Hammock, 2007; Dunn et al., 2011; Dunn \& Ellis, 2005; Han et al., 2008; Hasegawa et al., 2007). FTICR-MS was used to detect differences in metabolic profiles of rat urine due to amiodarone induced phospholipidosis (Hasegawa et al., 2007). The PCA score plots confirmed that amiodarone treated-rat urine was different in biochemical composition than control urine. About $20 \%$ of the detected ions were altered by more than 1.5 fold due to amiodarone dosing. Furthermore, the identities of three altered metabolites due to amiodarone treatment were confirmed (Hasegawa et al., 2007). Similarly, analysis of urine samples collected from 55 cancer patients using DFI-MS coupled to LTQ analyzer was able to confirm that body lean and fat mass in cancer patients have distinct metabolic profiles (Stretch et al., 2012). In conclusion, the widespread use of DIMS in urine metabolomics is currently being hampered by many technical and analytical challenges. However, with the continuously evolving research for improving its performance, an increase in the application of DIMS in urine metabolomics is anticipated in the near future.

University of Saskatchewan Author Manuscript, available April 16, 2017. 
This is the peer reviewed version of: Khamis, M. M., Adamko, D. J. and El-Aneed, A. (2015), Mass Spec Rev., published in final form at https://doi.org/10.1002/mas.21455.

\section{B. Gas Chromatography- Mass Spectrometry (GC-MS)}

The use of GC-MS is inherently biased towards low molecular weight volatile compounds making it well-suited for metabolomic analysis (Dunn et al., 2011). Unfortunately, the majority of metabolites lack sufficient volatility and therefore, chemical derivatization is employed to enhance their volatility (Dunn et al., 2011). In general, oximation followed by trimethylsilylation with various reagents such as bis-(trimethylsilyl)-trifluoroacetamide (BSTFA) convert metabolites bearing diverse functional groups (-COOH, $-\mathrm{NH}_{2},-\mathrm{OH},-\mathrm{SH}$ and others) into volatile products (Begley et al., 2009; Dunn et al., 2011; Fiehn et al., 2000; Halket et al., 2005; Villas-Bôas et al., 2011). However, this type of reactions consume longer times and produce lower stability products relative to the simpler alternative alkylation reactions with reagents as ethyl chloroformate (ECF) (Fig 4) (Dunn et al., 2011; Villas-Bôas et al., 2011).

GC-MS technique is generally used in urine metabolomic studies especially in the area of cancer biomarker discovery. A global metabolic profiling approach of urine using GC-ToF and UPLC-QToF identified 31 metabolites involved in bile acid, fatty acids, urea cycle and methionine metabolism as candidate diagnostic biomarkers in human hepatocellular carcinoma (HCC) (Chen et al., 2011b). Samples were collected from 177 participants (82 HCC patients, 24 benign liver tumor patients, 71 healthy controls). For GC-ToF, samples were diluted 1:1 with water (final volume $600 \mu \mathrm{L}$ ) and spiked with L-2-chlorophenylalanine as internal standard. Derivatization was achieved using ECF to yield volatile derivatives that were analyzed following LLE extraction (Chen et al., 2011b). It is worth to note that these metabolites, with the exception of threonine and hypoxanthine, were totally different than those detected by an earlier study which employed GC-MS coupled to a lower resolution mass analyzer, Quadrupole (Q), to differentiate between HCC and healthy participants (Wu et al., 2009). In the latter work, samples from 20 patients were used to identify 18 metabolites as potential diagnostic markers for HCC (Wu et al., 2009). A relatively larger urine volume $(1 \mathrm{~mL})$ was spiked with the aforementioned internal standard, filtered and derivatized with BSTFA (Wu et al., 2009). With the exception of 3 biomarkers, the differential expression of the detected metabolites was not justified nor their relevant metabolic pathways were illustrated (Wu et al., 2009). The significantly larger number of detected biomarkers in the first study (Chen et al., 2011b) can be attributed to its complementary use of two high resolution techniques. In addition, the larger sample size in the first study provided an additional benefit of enhanced statistical power.

In another work, a computational method was employed to identify nine genome-wide altered metabolic pathways in breast cancer (Nam et al., 2009). One hundred and twenty eight candidate biomarkers were selected. These metabolites were identified in the urine of 100 participants using GCMS and fifteen of them were further quantified. The authors were able to recognize 4 metabolites (homovanillate, 4-hydroxyphenylacetate, 5-hydroxyindoleacetate and urea) to be statistically different between healthy and breast cancer patients (Nam et al., 2009). GC-Q-MS was also used in an untargeted and targeted approaches to differentiate between breast, cervical and ovarian cancer patients using urine samples (Woo et al., 2009). Statistical analysis of the result in the untargeted approach could only distinguish breast cancer from the other two types. When combined to LC-MS method, the targeted approach was able to confirm the previously reported two nucleoside; 5-hydroxymethyl-2-deoxyuridine and 8-hydroxy-2-deoxyguanosine, as diagnostic biomarkers for breast cancer and suggested three new potential biomarkers for ovarian cancer (Woo et al., 2009).

To enhance the analytical performance of GC, two dimensional gas chromatography (GC×GC) coupled to MS was introduced. It is a relatively new technique that has been used in metabolomic 
This is the peer reviewed version of: Khamis, M. M., Adamko, D. J. and El-Aneed, A. (2015), Mass Spec Rev., published in final form at https://doi.org/10.1002/mas.21455.

studies providing superior sensitivity and resolving power in comparison to conventional GC-MS (Almstetter, Oefner \& Dettmer, 2012). A GC $\times$ GC-ToF method was able to target and quantify a complete set of biomarkers for six inherited metabolic disorders which was not achievable with a GC-QMS method (Wojtowicz et al., 2010). Recently, a GC $\times$ GC-ToF method was applied to an untargeted comprehensive evaluation of volatile metabolites in human urine (Rocha et al., 2012). Samples were extracted using solid phase micro extraction (SPME) prior to analysis. A total of 700 peaks were detected per sample, indicating the rich composition of urine for volatile compounds. Additionally, the volatile urine metabolome profiles obtained from smokers and non-smokers were also compared and differentiated. The investigation was possibly the most complete informative study covering the volatile metabolite component within the human urine (Rocha et al., 2012).

In conclusion, GC is the oldest hyphenated technique that has been used in combination with MS for over 50 years and it has been often described as the gold standard in metabolomics (Dunn et al., 2011; Dunn \& Ellis, 2005). Despite the continuous introduction of new and advanced techniques, GCMS is still considered as a highly attractive tool in metabolomics. This can be attributed to the numerous well-established and commercially available metabolite libraries and retention indices which are not readily available in LC-MS methods (Ryan et al., 2011). However, the implementation of lengthy prechromatographic derivatization and extraction steps to ensure analyte stability and volatility still constitute an unavoidable impediment to its wide applications (Dettmer, Aronov \& Hammock, 2007; Lei, Huhman \& Sumner 2011; Theodoridis, Gika \& Wilson, 2011; Theodoridis et al., 2012). Such sample pre-treatment often introduces variability as well derivatization artifacts to the analyzed samples which complicate data interpretation (Dettmer, Aronov \& Hammock, 2007; Johnson \& Gonzalez, 2012; Lei, Huhman \& Sumner 2011; Theodoridis et al., 2012; Villas-Bôas et al., 2011). Furthermore, it was reported that silylation can results in conversion reactions such as the conversion of arginine into ornithine (Halket et al., 2005). However, the inherent analytical advantages of GC-MS as high sensitivity, reproducibility, and resolution power outweigh its associated problems and endorse it as a cornerstone tool in the complete analysis of the urine metabolome (Johnson \& Gonzalez, 2012; Scalbert et al., 2009; Theodoridis, Gika \& Wilson, 2011; Theodoridis et al., 2012).

\section{Capillary Electrophoresis- Mass Spectrometry (CE-MS)}

In CE-MS, charged metabolites are separated based on their differential migration velocities in an electrically conductive liquid phase under the influence of an external electric current (Bayer, 2001; Dunn et al., 2011). CE-MS exhibits comparable or better separation efficiency than GC-MS and LC-MS in terms of shorter analysis times. However, CE-MS is less frequently used in urine metabolome analysis due to its lower sensitivity and its poor reproducibility in both peak area and migration time. Such inconsistencies are caused by the sorption of macromolecules in urine onto the capillary wall (Dunn et al., 2011; Lei, Huhman \& Sumner 2011; Theodoridis, Gika \& Wilson, 2011). Therefore, in comparison to GC-MS and LC-MS, the number of investigations employing CE-MS in urine metabolome analysis is limited, with more applications in fingerprinting rather than the quantitative targeted platform (Ramautar et al., 2011; Ramautar, Somsen \& de Jong, 2009, 2013). For example, a CE-ToF method was developed for the profiling and the targeted analysis of free estrogens and their glucuronide and sulfate conjugates (Kuehnbaum \& Britz-McKibbin, 2011). In spite of adequately resolving free and conjugated estrogens in alkaline buffer, the method could not detect endogenous estrogens present in sub-micromolar concentrations in human urine due to inadequate sensitivity. Moreover, the incorporation of an additional SPE step resulted in the removal of the conjugated forms from enriched premenopausal female urine extracts (Kuehnbaum \& Britz-McKibbin, 2011). 
This is the peer reviewed version of: Khamis, M. M., Adamko, D. J. and El-Aneed, A. (2015), Mass Spec Rev., published in final form at https://doi.org/10.1002/mas.21455.

CE-MS is more suited for the separation of cationic species over anionic compounds. For the latter group, modifications can be done to the capillary through coating with cationic surfactants (Yang, Regnier, \& Adamec, 2008). However, cationic surfactants are subjected to alkaline hydrolysis causing the creation of voids within the separation phase and subsequent current drop (Soga et al., 2003). An alternative simpler approach is to modify the physical and chemical properties of anions through derivatization with a positively charged derivatizing agent. Profiling of carboxylic acid-containing metabolites in rat urine using CE-MS and CE-MS/MS was feasible through using N-butyl-4aminomethylpyridinum iodide (BAMP) and N-hexyl-4-aminomethyl-pyridinum iodide (HAMP) reagents (Fig 5) (Yang, Regnier, \& Adamec, 2008). After derivatization, positively charged compounds were produced and analyzed through normal CE mode. Analysis of rat urine revealed 59 candidate ions matching the characteristic pattern of carboxylic acids, out of which, only the identity of 32 was confirmed (Yang, Regnier, \& Adamec, 2008).

CE-MS was also applied for the analysis of polypeptides in urine. Polypeptides serve as an important biomarker group for renal diseases. A CE-ToF method was utilized in screening urine samples obtained from patients suffering from kidney disease and healthy individuals (Wittke et al., 2003). A healthy urine pattern consisting of 247 polypeptides was established and it was found to be distinctively different from that of the diseased patients. Twenty seven polypeptides, that were not present in the healthy patterns, were present in more than $50 \%$ of urine samples obtained from the patient population. In addition, thirteen of the normal polypeptides were absent in the urine of these patients (Wittke et al., 2003).

\section{Liquid Chromatography- Mass Spectrometry (LC-MS)}

LC, coupled to different detection techniques, is the most widely employed platform in urine analysis. Combined with the qualitative and quantitative capabilities of MS, and due to the continuous improvements in its related technologies (i.e. UPLC, Hydrophilic interaction chromatography (HILIC)), LC-MS is ideally suitable for metabolomics studies, where it can be tailored for targeted or untargeted purposes (Ryan et al., 2011; Theodoridis, Gika \& Wilson, 2011; Zhang et al., 2012a). In general, RP stationary phase is well suited for the separation of non-polar and polar metabolites in urine (Dettmer, Aronov \& Hammock, 2007; Lei, Huhman \& Sumner 2011). However, when highly polar low molecular weight metabolites are analyzed, they are usually eluted near or at the void volume with poor reproducibility (Dettmer, Aronov \& Hammock, 2007; Pesek et al., 2008; Zhang et al., 2012c). Therefore, aqueous normal phase (ANP) is a convenient alternative for such compounds, where silica hydride-based high-pressure liquid chromatography (HPLC) stationary phases separate metabolites through a dual retention mechanism of hydrophilic and hydrophobic interactions (Pesek et al., 2008; Zhang et al., 2012c). RP and ANP in combination with QToF and QqQ-MS were utilized for the global metabolite mapping and discovery of potential biomarkers of bladder cancer (Putluri et al., 2011). By adopting non-targeted and targeted approaches, 25 metabolites were identified suggesting their aptitude for further investigation as diagnostic and prognostic biomarkers of bladder cancer (Putluri et al., 2011).

HILIC is another suitable technology for the separation of ionic-polar metabolites (Buszewski \& Noga, 2012; Chen et al., 2009; Ryan et al., 2011; Want et al., 2010). HILIC is a hybrid between normal phase (NP)-LC and RP-LC systems. Similar to NP-LC, polar stationary phases such as silica or derivatized silica (amino or cyano) are used allowing for the separation of polar metabolites that cannot be retained on RP-LC columns. Meanwhile, HILIC shares the use of water-containing polar organic mobile phases similar to RP-LC. Therefore, HILIC solves the problem of the limited solubility of polar metabolites that cannot be separated using NP-LC mobile phases (Buszewski \& Noga, 2012). A 
This is the peer reviewed version of: Khamis, M. M., Adamko, D. J. and El-Aneed, A. (2015), Mass Spec Rev., published in final form at https://doi.org/10.1002/mas.21455.

comparative evaluation of the metabolome profiles of rat urine using HILIC and RP columns utilizing UPLC-QToF system revealed that the two separation technologies provide complementary data leading to a better metabolome coverage (Gika, Theodoridis, \& Wilson, 2008a). The use of C18 column (i.e. RP chromatography) produced data containing a larger number of observed peaks with enhanced resolution. On the other hand, sensitivity was enhanced with the HILIC column which could be attributed to the superior ionization desolvation process resulting from the higher organic composition of the mobile phase needed for the elution of the well-retained compounds (Gika, Theodoridis \& Wilson, 2008a). In general terms, HILIC can explore different portions of the urine metabolome giving data on "MS friendly" zwitterion as well as highly polar metabolites such as taurine, betaine, creatine, creatinine and carnitine (Gika, Theodoridis \& Wilson, 2008a; Lei, Huhman \& Sumner 2011; Trivedi \& Iles, 2014b; Trivedi et al., 2012; Zhang et al., 2012c). Both UPLC-RP and HILIC were employed to screen the urine metabolic profiles from liver cancer patients and healthy participants using QToF (Chen et al., 2009). As predictable, compared to RPLC-MS, HILIC-MS produced more sensitive response allowing for a better separation of the two groups. However, RPLC-MS was useful in separating compounds related to fatty acid oxidation as acylcarnitines. Out of the 21 identified potential biomarkers, the HILIC mode was able to detect 16 metabolites. In addition, it was able to retain and separate some of the hydrophobic acylcarnitines that were analyzed by RPLC due to the presence of quaternary ammonium group within their structures (Chen et al., 2009).

In addition to column technologies, the introduction of UPLC has resulted in significant improvements in urine metabolome analysis (Dettmer, Aronov \& Hammock, 2007; Wang et al., 2011). The performance of conventional HPLC to UPLC was compared using orthogonal acceleration-ToF (oaToF) in the profiling of urine obtained from three different mouse strains (Fig 6) (Wilson et al., 2005). Using UPLC, method sensitivity was enhanced by 3-5 folds while providing 10 folds faster analyses. Sharper and distinct peaks were also obtained with substantial improvement in resolution which is reflected by the number of detected metabolites (13,000 in UPLC vs. 2,000 in HPLC) (Wilson et al., 2005). Similarly, the performance of a UPLC-oaToF-MS method developed for high throughput discrimination of five rodent strains according to age, gender, strain and diurnal variation (Plumb et al., 2005) was compared to a previously developed HPLC-ToF method (Plumb et al., 2003). According to the authors, 3,900 marker ions were detected in a 1.5 min run time using UPLC-oaToF in comparison to merely 1,000 ions detected in the 10 min run of HPLC-QToF (Plumb et al., 2005; Plumb et al., 2003).

\section{LC-MS QUANTITATIVE APPROACHES}

\section{A. Stable Isotope Labeling (SIL)}

Following identification in the untargeted approach, candidate metabolites must be quantified in urine for the selection of potential biomarkers using various statistical tools. However, when ESI is used as the ionization source, ion suppression from co-eluting molecules and matrix interference is a major impediment in MS quantitative analysis (Gangl et al., 2001; Guo \& Li, 2009; Ryan et al., 2011; Stokvis et al., 2004; Toyo'oka, 2012). Therefore, regardless of the used MS- based technique, quantification of metabolites in the targeted approach cannot be achieved through external standard (ES) calibration and the use of internal standards (IS) becomes inevitable (Dunn et al., 2011). Structural analogues, when used as IS, might elute at different times than the compounds of interest and would consequently experience different matrix effects leading to insufficient accuracy and precision (Guo, Ji \& Li, 2007; Ryan et al., 2011; Stokvis et al., 2004). One strategy to overcome such hurdles is the use of an isotopically labeled internal standard (i.e. stable isotope labeling, SIL) (Guo \& Li, 2009; Jacob et al., 2002; Ryan et al., 2011; Toyo’oka, 2012). For example, the analytical performance of an HPLC-QqQ 
This is the peer reviewed version of: Khamis, M. M., Adamko, D. J. and El-Aneed, A. (2015), Mass Spec Rev., published in final form at https://doi.org/10.1002/mas.21455.

for the determination of 1-hydroxypyrene (a biomarker of polycyclic aromatic hydrocarbon exposure) in urine using two calibration modes was evaluated (Pigini et al., 2006). Blank urine was spiked either with external reference standard of 1-hydroxypyrene or with 1-hydroxypyrene- $\mathrm{d}_{9}$ as an IS. The analytical performance of the methods regarding linearity, precision and accuracy did not significantly differ between the two calibration strategies. Such findings were confined to when the same urine was used for both calibrations, and therefore the matrix effect was unified. However, it was confirmed that external calibration cannot be used in routine biomarker analysis where, logically, urine samples from different subjects are investigated (Pigini et al., 2006).

Although efficient in overcoming matrix effect, the use of SIL is well suited when a single (Jacob et al., 2002; Kuklenyik, Ashley \& Calafat, 2002) or few compounds (Clarke et al., 2002; Weimann, Belling, \& Poulsen 2002; Xu et al., 2005) are to be traced in urine. However, when large numbers of urinary metabolites are targeted, SIL approach is expensive and impractical due to the structural diversity of metabolites (Lei, Huhman \& Sumner 2011; Toyo'oka, 2012). Furthermore, it has been shown that the addition of a large number of ISs may interfere with the detection of the investigated metabolites (Hodson et al., 2009).

\section{B. Differential Isotope Labeling (DIL)}

Another relatively new approach in metabolomics is the use of DIL where metabolites are targeted based on their common functional groups. An isotope labeled derivatizing reagent introduces an isotope tag to the investigated metabolite group within a urine sample. An isotopically-labeled analogue of the same reagent, commonly containing Deuterium (D) or ${ }^{13} \mathrm{C}$ (i.e. heavy reagent), is used for the derivatization of a second comparable urine sample or standard solution. Finally, equal aliquots from light and heavy labeled solutions are mixed for relative or absolute quantification (Fig 7) (Guo \& Li, 2009; Toyo'oka, 2012). It is worth to note that there is a vast number of derivatizing reagents that proved to be useful in LC-MS analysis (Santa, 2013), however, Table 1 summarizes the most important reagents that were employed in metabolomic using urine as a biofluid.

Guo K et al (Guo, Ji \& Li., 2007) developed a ${ }^{13} \mathrm{C}$ and deuterated-isotope dimethylation labeling reaction for the quantification of primary and secondary amine containing metabolites in urine through reductive amination. One concern regarding this reaction is that it resulted in minimum increase in hydrophobicity of polar metabolites through the formation of the dimethylated (primary amine) or monomethylated (secondary amine) derivatives. Consequently, the integrative use of HILIC and RP-LC was required for the analysis of "highly polar" and "less polar" metabolites, respectively, within each sample. In addition, despite providing a 4-Da mass difference with the deuterium dimethylated-labeled species, deuterated metabolites eluted at marginally different times than non-deuterated ones and the use of HILIC was fundamental to overcome such discrepancies (Guo, Ji \& Li., 2007). The difference in the elution time is mainly due to the variance between $\mathrm{C}-\mathrm{H}$ and $\mathrm{C}-\mathrm{D}$ bond properties leading to differences in the interaction with the hydrophobic stationary phase. As a result, the isotope counterparts are subjected to different matrix and ion suppression effects (Turowski et al., 2003; Wade, 1999). To address such concern, the use of ${ }^{13} \mathrm{C}$ labeling can theoretically eliminate elution issues related to the use deuterium for labeling. However, in case of ${ }^{13} \mathrm{C}$-methylation, light and heavy-labeled secondary amines exhibit only a mass difference of $1 \mathrm{Da}$, limiting the applicability of such labeling to merely high resolution MS instruments (Guo, Ji \& Li., 2007).

Due to the aforementioned drawbacks, another differential ${ }^{12} \mathrm{C}-/^{13} \mathrm{C}$-isotope dansylation labeling reaction was developed and resulted in significant improvements in the profiling of phenol and aminecontaining metabolites in urine (Fig 7) (Guo \& Li, 2009; Zheng \& Li, 2012). It was shown that the 
This is the peer reviewed version of: Khamis, M. M., Adamko, D. J. and El-Aneed, A. (2015), Mass Spec Rev., published in final form at https://doi.org/10.1002/mas.21455.

introduction of a dimethyl amino naphthalene moiety resulted in; better retention of polar metabolites during LC analysis, enhancement of the ionization efficiency and improvement of signal intensity and signal-to-noise ratio. A dansylated library of 121 metabolites was constructed and used for the absolute quantification of 93 metabolites (Guo \& Li, 2009).

Recently, the organic acid urine sub-metabolome was analyzed through dimethylaminophenacyl differential labeling (Guo \& Li, 2010; Peng \& Li, 2013). The derivatized compounds resulted in 101000 folds increase in signal intensity in comparison to their underivatized counterparts (Peng \& Li, 2013). Diverse DIL reactions were also developed to tag urinary metabolites bearing other chemical functional groups. For instance, thiols and disulfides were quantified in human urine through two sequential labeling reactions with two ferrocene-based maleimide reagents (Table 1) (Seiwert \& Karst, 2007). Furthermore, steroids containing a hydroxyl group were derivatized with deuterium 4(dimethylamino)-benzoic acid (d4-DMBA) (Table 1) (Dai et al., 2012). Through D/H isotope DMBA tagging, twenty one steroidal hormones were relatively quantified in males, females and pregnant females urine using LC-LTQ-Orbitrap preceded by an SPE extraction step for the removal of abundant polar metabolites (Dai et al., 2012).

In addition to overcoming ion suppression and matrix effects that hinder metabolite quantification, DIL adds other unique benefits in metabolome analysis. The additional hydrophobic tag allows polar metabolites to be retained on RP columns; therefore, their separation is enhanced. Higher organic phase is also needed for their elution, thus enhancing the ionization efficiency and sensitivity (Dunn et al., 2011; Eggink et al., 2010). Similarly, LC analysis of volatile compounds with limited stability, such as aldehydes, is challenging which can be addressed using DIL approach (Eggink et al., 2010). The usefulness of 4-(2-((4-bromophenethyl) dimethylammonio)ethoxy)benzenaminium dibromide (4-APEBA) (Eggink et al., 2010) as a novel aldehyde derivatizing reagent was compared to the older reagent; 4-(2-(trimethylammonio)ethoxy) benzeneaminium dibromide (4-APC) (Table 1) (Eggink et al., 2008). The presence of the additional bromophenethyl group in the former reagent provided several benefits over the older generation. First, the increase in the molecular weight resulted in improvements in signal-to-noise ratio with 2 folds increase in the response of the derivatized products. Second, the new reagent reacted with carboxylic acid containing compounds as well, thus increasing its potentials as a suitable reagent for aldehydes and acids sub metabolome profiling. Third, the isotopic signature of ${ }^{79} \mathrm{Br} /{ }^{81} \mathrm{Br}$ produced distinct MS/MS fragmentation patterns facilitating identification (Eggink et al., 2010).

\section{LC-MS IN URINARY BIOMARKER DISCOVERY}

LC-MS has an indispensable role in exploring new diagnostic biomarkers (Roux et al., 2011; Zhang et al., 2012b), specifically, in the area of cancer as demonstrated by the previously discussed examples (Chen et al., 2009; Chen et al., 2011b; Woo et al., 2009). Due to the complexity of this disease, continuous studies are being conducted with the ultimate target of providing biomarkers that are non-invasively collected for early detection of different cancer types. In an attempt to quantify altered nucleosides in urogenital cancer, twelve nucleosides were analyzed using HPLC-QqQ-MS in the urine of healthy and urogenital cancer participants. Five nucleosides, namely; N-2-methylguanosine, 3methyluridine, 6-methyladenosine, inosine, and N,N-dimethylguanosine were found to be statistically different between the two groups (Struck et al., 2013). Despite this finding, it is difficult to ascertain that a specific nucleoside is a biomarker for a specific cancer type as the urinary levels of nucleosides are generally elevated in cancer patients due to the higher RNA turnover (Schram, 1998). In fact, some of 
This is the peer reviewed version of: Khamis, M. M., Adamko, D. J. and El-Aneed, A. (2015), Mass Spec Rev., published in final form at https://doi.org/10.1002/mas.21455.

the identified nucleoside metabolites were also found to be differentially expressed between healthy and individuals having other types of cancer (Cho et al., 2009; Hsu et al., 2011; Jeng et al., 2009).

As for renal cell carcinoma (RCC), a combination of UPLC-LTQ-MS and GC-MS methods established a signature urinary profile (Kim et al., 2011). Three biomarkers were differentially expressed in urine of RCC patients, where the concentrations of 4-hydroxybenzoate and gentisate decreased and that of quinolinate increased compared to control urine. However, the study was not able to confirm whether the three metabolites were specific only to RCC or to any kind of cancer in general. Further investigations involving global metabolome analyses and larger sample size were suggested before the use of the aforementioned metabolites as diagnostic biomarkers for RCC (Kim et al., 2011).

In addition to cancer, biomarkers for other diseases have been investigated using LC-MS. A UPLC-QToF method was used for the non-targeted analysis of urine samples collected from patients with jaundice syndrome (Wang et al., 2012). Complete separation of patients from healthy individuals was achieved using 44 metabolites. Such metabolites were suggested to be further explored for the development of a clinically useful diagnostic tool (Wang et al., 2012).

Another example is children who undergo cardiopulmonary bypass surgery (CPB). They are prone to the complication of acute renal injury (AKI) with a mortality rate over 50\% (Beger et al., 2008; Williams et al., 2002). A UPLC-ToF method was developed for profiling urine samples obtained from 40 children before and after CPB surgery (Beger et al., 2008). Patients developing AKI were well separated from the other group using PCA score plots. One potential biomarker suggested by PCA was further identified by MS/MS as homovanillic acid sulfate (HVA-SO ${ }_{4}$ ). Only $4 \mathrm{~h}$ following surgery, the concentration of $\mathrm{HVA}-\mathrm{SO}_{4}$ was doubled in patients who developed AKI while slightly increased in patients without AKI. Therefore, HVA can serve as a potential biomarker allowing for early detection of AKI in this vulnerable population.

Urinary metabolomics can also be useful in understanding complex psychological disorders such as depression (Zheng et al., 2010). A UPLC-QqQ method was used in the metabolic profiling of urine obtained from 16 healthy and chronic unpredictable mild stress model rats. Using PCA, complete separation between the two groups was observed in the positive and negative ion modes. In addition, six potential biomarkers related to energy metabolism, amino acid metabolism and gut microflora were identified (Zheng et al., 2010). Such detections highlight the potential of MS-based metabolomics for the future discovery of new urinary biomarkers suitable for the clinical diagnosis of complex psychological and neurological disorders.

\section{INTEGRATIVE USE OF MS-BASED PLATFORMS FOR HOLISTIC URINE METABOLOME ANALYSIS}

The comprehensive analysis of metabolites within urine is, unfortunately, a technical challenge due to their diversity in physiochemical properties, concentrations, stabilities and excretion patterns. Mass spectrometric platforms such as LC-MS, GC-MS, DIMS, and CE-MS have become indispensable tools in system biology analysis due to their inherent sensitivity, specificity and quantitative capabilities. However, each of the aforementioned techniques has its own advantages and limitations concerning sensitivity, separation efficiency and cost. Therefore, the best strategy for holistic metabolome visualization is, logically, through the application of complementary analytical techniques (Dunn, 2008; Dunn et al., 2011; Dunn \& Ellis, 2005; Kumar et al., 2012; Lei, Huhman \& Sumner 2011; Woo et al., 2009). For example, in an attempt to discover urinary biomarkers for hepatotoxicity, rats were orally dosed with carbon tetrachloride, acetaminophen and methotrexate (Kumar et al., 2012). Global profiling 
This is the peer reviewed version of: Khamis, M. M., Adamko, D. J. and El-Aneed, A. (2015), Mass Spec Rev., published in final form at https://doi.org/10.1002/mas.21455.

of urine using UPLC-QToF followed by partial least square discriminate analysis revealed altered urinary patterns and the score plots of healthy and treated rats were separately clustered. Some metabolites belonging to three different metabolic pathways were selected as candidate hepatotoxicity biomarkers and these were classified into; steroids, bile acids and amino acids (Kumar et al., 2012). Urinary steroids were quantified using a previously published GC-MS method (Moon et al., 2009). It was found that urinary concentrations of $11 \beta-\mathrm{OH}$-androsterone, epiandrosterone and estrone decreased, and that of 11-dehydrocorticosterone increased significantly following hepatotoxicity. As for bile acids and oxysterols, only cholic acid and lithocholic acid were differentially overexpressed in the urine of treated groups analyzed by another GC-MS method (Kumar et al., 2011). Finally, 25 amino acids that were differentially expressed in urine of the three treated groups were quantified using a CE-MS (Shama et al., 2008) and six compounds were selected as hepatotoxicity biomarkers.

Six different platforms were also employed in order to widen the systematic characterization of the human urinary metabolome (Bouatra et al., 2013). This holistic approach led to the identification of 445 and quantification of 378 unique metabolites. Four different GC-MS methods were applied to target bile acids, volatile compounds, organic acids and polar metabolites. In total, all methods led to the identification of 179 and quantification of 85 metabolites. A combined DFI-MS/MS and LC-MS/MS method led to the identification and quantification of 127 metabolites. Furthermore, quantitative results of 40 metals or trace elements were achieved by inductively coupled plasma (ICP)-MS.

\section{MS DATA MINING AND STANDARDIZATION IN URINE METABOLOME ANALYSIS}

Enormous amounts of complex data are generated following the untargeted MS analysis of the urine samples, and in its current format, these data cannot be manually analyzed (Monteiro et al., 2013). Therefore, the first critical step in data mining is to correct all variations caused by the experimental variables. Data handling (including; peak normalization, peak alignment, baseline correction and peak deconvolution) is usually achievable through the use of commercially available software (Monteiro et al., 2013; Zhou et al., 2014). Subsequently, multivariate statistical techniques (supervised and unsupervised) are performed to allow for the recognition of patterns between samples (i.e. between healthy and diseased patient's urine) (Monteiro et al., 2013). As a final step, differentially expressed metabolites must be identified through the available databases before a targeted approach can be adopted (Dunn et al., 2011; Monteiro et al., 2013). Data analysis and interpretation is a fundamental step in metabolomic studies that may lead to erroneous results. However, this side of data handling is mainly involved with software manipulation rather than urine itself. Therefore, the reader is advised to refer to the literature for more comprehensive understanding of this topic (Katajamaa \& Orešič, 2007; Monteiro et al., 2013; Scalbert et al., 2009).

However,one concerning variable in data pre-processing that lies within the scope of this work is the variation in urine volume. . In fact, in addition to the complexity of urine metabolome composition, fluctuation in urine volume represents another analytical challenge as it results in erratic dilutions in metabolite concentrations. Urine volume varies from 600 to $2500 \mathrm{~mL} /$ day depending on the individual's hydration status, which in turn is affected by fluid intake, humidity, temperature and physical activity (Hee, 1993; Ryan et al., 2011). Therefore, regardless of the analytical platform, mathematical manipulations of the analytical raw data must be done to normalize concentrations of metabolites to other urine properties with lower extend of variation than urine volume. Among the most common normalization strategies are the creatinine concentration, specific gravity and osmolality (Hee, 1993; Ryan et al., 2011). 
This is the peer reviewed version of: Khamis, M. M., Adamko, D. J. and El-Aneed, A. (2015), Mass Spec Rev., published in final form at https://doi.org/10.1002/mas.21455.

In the absence of kidney diseases, creatinine is excreted in measurable and relatively constant amounts by glomerular filtration into the urine (Boeniger, Lowry \& Rosenberg, 1993; Hee, 1993; Viau, Lafontaine \& Payan, 2004; Warrack et al., 2009). In general, it is accepted that creatinine has minimal metabolic or excretory variances among healthy individuals (Adamko et al., 2007; Ryan et al., 2011). In other words, the amount of creatinine excreted per day is constant and approximately at a rate of $1 \mathrm{~g}$ per $20 \mathrm{Kg}$ of muscle mass ( Viau, Lafontaine \& Payan, 2004). Creatinine's rate of elimination is independent of urine flow and therefore, its concentration is inversely proportional to urine volume (Boeniger, Lowry \& Rosenberg, 1993). Therefore, creatinine is accepted to behave as the best internal standard for correcting urine volume variations as the ratio of creatinine to the analyte of interest is maintained in urine (Adamko et al., 2007; Ryan et al., 2011). Despite being the most employed normalization technique, the suitability of creatinine, however, has always been debated due to the presence of various uncontrollable factors influencing its concentration such as age, gender, physical activity, time of the day and food intake (Boeniger, Lowry \& Rosenberg, 1993; Ryan et al., 2011). Maintaining proper hydration and normal meat intake during sample collection can contribute to minimizing this variation ( Boeniger, Lowry \& Rosenberg, 1993).

Normalization to specific gravity is an alternative methodology that uses the mathematical equation developed by Levine and Fahy (Levine \& Fahy, 1945) to correct the measured specific gravity of fresh urine to a mean specific gravity reference value, usually of 1.02 (Chadha, Garg \& Alon, 2001; Cone et al., 2009; Heavner et al., 2006; Hee, 1993). However, like creatinine normalization, this method has several limitations. For instance, urine density is affected by the total mass of the solutes, which in turn is affected by their number and molecular weight. Therefore, the specific gravity method is not applicable in cases such as diabetes mellitus where high glucose concentration is excreted in urine as well as kidney patients with urine containing high protein content (Chadha, Garg \& Alon, 2001; Miller et al., 2004). Furthermore, the mean specific gravity of normal urine is not constant and it has a range of 1.016 to 1.024 (Heavner et al., 2006). Finally, urine density is affected by fluctuating temperature as well as the hydration state of the patient (Chadha, Garg \& Alon, 2001; Miller et al., 2004).

Similar to specific gravity, osmolality measures the total solute concentration in urine and it is determined by the depression in the solution's freezing point or vapor pressure (Chadha, Garg \& Alon, 2001). In contrast to creatinine and specific gravity, osmolality is not affected by diurnal rhyme, diet, activity, age, gender, stress or mass, charge or size of particles (Chadha, Garg \& Alon, 2001; Warrack et al., 2009). In fact, the determination of urine osmolality gives the most specific reflection of its metabolite concentration, however, this technique is not readily accessible in all laboratories (Chadha, Garg \& Alon, 2001). Consequently, measuring specific gravity is usually implemented as it can be converted mathematically into an approximate corresponding osmolality value (Chadha, Garg \& Alon, 2001).

A new normalization approach for amine and phenol containing metabolites using chemical labeling reaction was recently reported $(\mathrm{Wu} \& \mathrm{Li}, 2012)$. Urine samples were derivatized with dansyl chloride and analyzed by a very fast LC-UV method. The total concentration of all co-eluted labeled metabolites in each urine sample was calculated. The same sample was then analyzed by an LC-FTICRMS method and each selected metabolite was quantified using heavy and light dansyl chloride labeling and then normalized to the total concentration ( $\mathrm{Wu} \& \mathrm{Li}, 2012)$. Although that the superiority of such normalization approach was clearly demonstrated over the creatinine and the osmolality approaches, the dansyl chloride method, however, requires the tedious steps of derivatization and running an additional LC-UV for the calculation of total labeled metabolite concentrations. In addition, it confines the 
This is the peer reviewed version of: Khamis, M. M., Adamko, D. J. and El-Aneed, A. (2015), Mass Spec Rev., published in final form at https://doi.org/10.1002/mas.21455.

quantification to these metabolites bearing only primary or secondary amine and phenolic functional groups that can react with the designated reagent.

Another new global normalization approach was developed using a customized software based on MS total useful signal "MSTUS" (Warrack et al., 2009). Untargeted urine analysis was first achieved using LC-LTQ-FTICR-MS. Subsequently, all signals present in the spectra of all samples were summed and used for the generation of MSTUS normalization factor. This strategy has the advantage of eliminating the interferences from xenobiotics and artifacts (Warrack et al., 2009).

It is clear that despite the availability of different approaches for normalization, each has its own unavoidable limitations. For that reason, the application of two normalization strategies would definitely minimize the errors induced by either strategy and hence, confirm the changes detected within a urine metabolomic profile.

\section{CONCLUDING REMARKS}

Metabolomics research has intensified over the past decade as metabolites are the final output for biochemical activities within cells, providing an attractive platform for biomarker discovery. Therefore, intense research has been conducted to link a disease state to a specific set of metabolites that can be used for diagnosis (Beger et al., 2008; Kim et al., 2011; Wang et al., 2012; Woo et al., 2009) or for monitoring disease progression (Pasikanti et al., 2010; Sreekumar et al., 2009) or response to therapy (Hasegawa et al., 2007; Kumar et al., 2010; Van Doorn et al., 2007). While any biological fluid can be used for the identification of clinically-significant biomarkers, urine is an ideal option as it can be collected non-invasively with no discomfort to patients. Urine in particularly is an excellent choice for patients who cannot easily provide blood samples, such as children. However, little research has been performed regarding the stability of metabolites within urine after collection. Most concerning is the conflicting reports regarding such stability (Dunn et al., 2008; Gika, Theodoridis, \& Wilson, 2008a; Lauridsen et al., 2007; Sykes, 2007). It is, therefore, advisable that urine samples are aliquoted upon collection and stored at $-80^{\circ} \mathrm{C}$ till the time of analysis.

While many analytical tools can be used for the identification and quantification of urine metabolites, mass spectrometry, especially when linked to a suitable separation technique, can serve as an ideal platform for metabolomics research (Armitage \& Barbas, 2014; Dunn et al., 2013; Trivedi \& Iles, 2014a; Zhang et al., 2012a). MS offers the preferred sensitivity, high throughput capability and the desired quantitative outputs, absolute or relative. However, due to the versatile nature of metabolites, none of the currently available MS techniques can provide a single comprehensive characterization of any biofluid's constituents, including the urine. Indeed, reports providing highly informative metabolic pictures usually involve the integrative implementation of two or more MS-based platforms (Bouatra et al., 2013; Chen et al., 2011b; Kim et al., 2011).

Current MS-based research focuses on either developing identification/quantification methods for a subset of the metabolome based on the chemical structure (Guo \& Li, 2009, 2010; Rijk et al., 2009; Struck et al., 2013) or the identification of a set of diagnostic biomarkers linked to a specific physiological condition such as cancer (Nam et al., 2009; Struck et al., 2013). Unlike genes or proteins, the key challenge for metabolites is their diverse chemical structure. Recently, a subset of the urine metabolome was linked to asthma and COPD lung diseases using NMR (Saude et al., 2009; Saude et al., 2011). The next step is to quantify the target metabolites using MS platforms. Due to the structural diversity of the identified metabolites, multiple MS-based methods (some of which require 
This is the peer reviewed version of: Khamis, M. M., Adamko, D. J. and El-Aneed, A. (2015), Mass Spec Rev., published in final form at https://doi.org/10.1002/mas.21455.

derivatization) should be employed to obtain reliable quantitative data. Such a task is an example of one of the key challenges in MS-based metabolomics research.

Another concern is the actual identification of the observed ions within an MS run. Many investigations simply report the number of identified peaks (sometime in the thousands range) without actually identifying the molecular structures of these peaks (Peng \& Li, 2013; Rocha et al., 2012; Wilson et al., 2005). Even when differentially-expressed ions are identified in terms of structure, the underlying biochemical pathways that led to their relative increase/decrease are rarely rationalized. Such challenge is maximized with the fact that MS methods are designed to target a subset of the metabolome while the biochemical pathway that is linked to a specific physiological state is most likely complex. The current state of MS-based metabolomics probably resembles the infancy state in which MS-based proteomics existed twenty years ago.

In summary, it is clearly evident that MS metabolomics including urine-based is still at its infancy. More efforts are needed before validated sets of urinary biomarker metabolites can be routinely analyzed in clinical laboratories for the diagnosis and prognosis of complex diseases. However, the continuous development and merging of new and advanced high throughput hyphenated MS-based analytical techniques with strong bioinformatics tools will eventually allow for better interpretation of the metabolic data and wider understanding of the biochemical process occurring in the body. Assertively, the generated data must be shared in a way to permit use and re-use, however, a limited number of research groups freely provide their data to the scientific community. In 2005, the Metabolomics Standards Initiative (MSI) was launched with the goal of developing and communicating standards for the metabolomic community. The main aim within MSI group is to generate a broad community consensus around the proposed metabolomics standards so that the data can be available to others for evaluation or to support new research initiatives (Dunn et al., 2011; Fiehn et al., 2007). 
This is the peer reviewed version of: Khamis, M. M., Adamko, D. J. and El-Aneed, A. (2015), Mass Spec Rev., published in final form at https://doi.org/10.1002/mas.21455.

TABLE 1. Derivatizing reagents employed in urinary metabolites determination

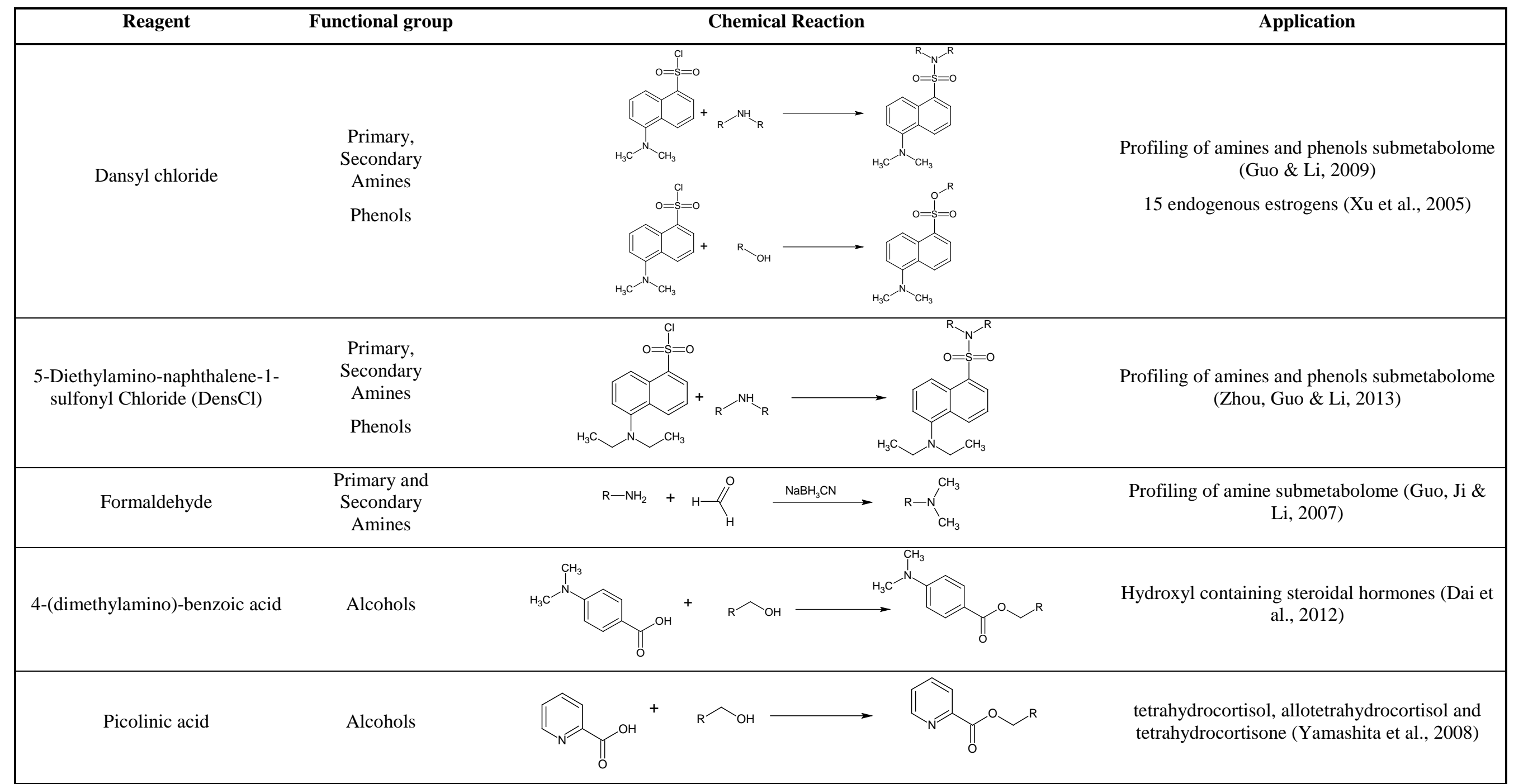


This is the peer reviewed version of: Khamis, M. M., Adamko, D. J. and El-Aneed, A. (2015), Mass Spec Rev., published in final form at https://doi.org/10.1002/mas.21455.

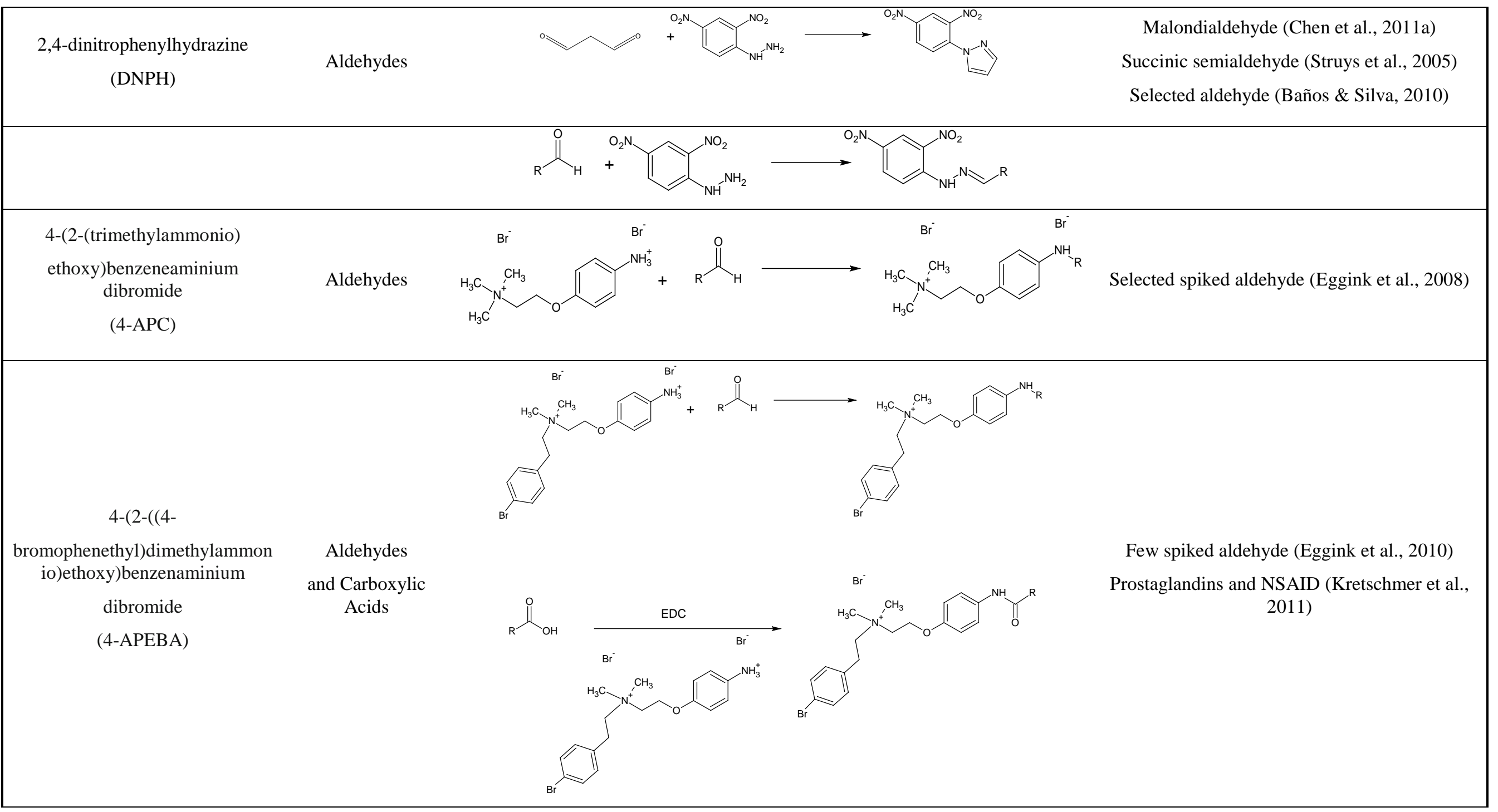


This is the peer reviewed version of: Khamis, M. M., Adamko, D. J. and El-Aneed, A. (2015), Mass Spec Rev., published in final form at https://doi.org/10.1002/mas.21455.

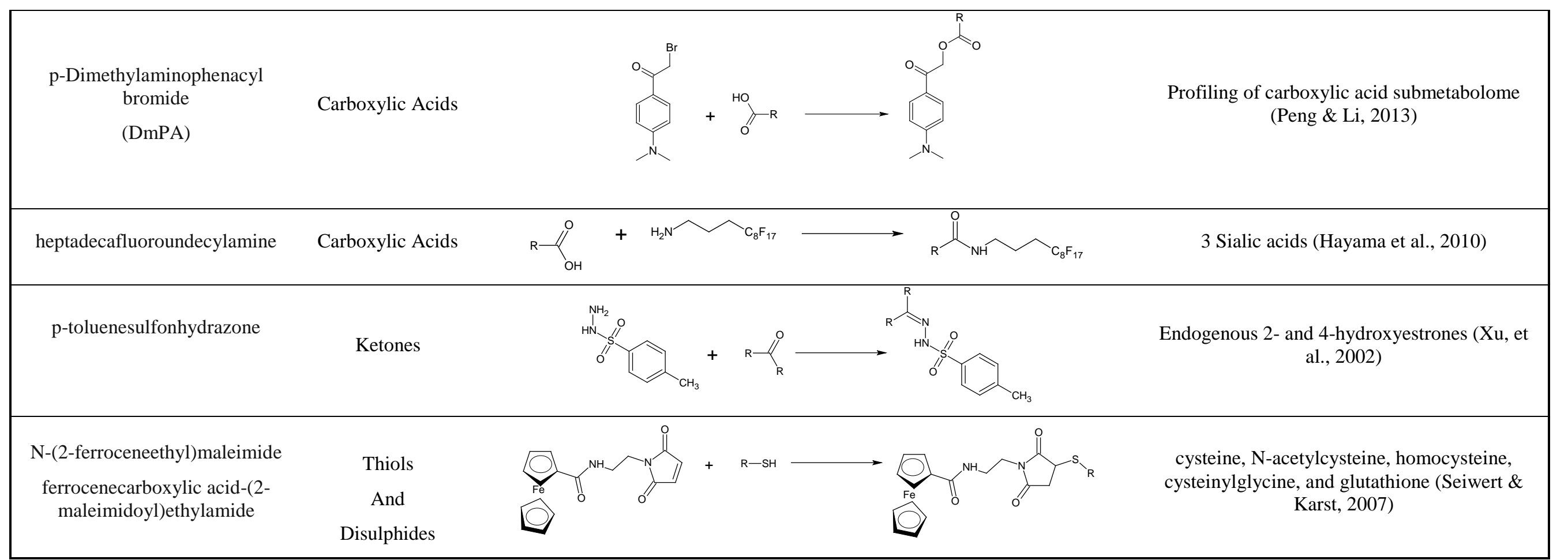


This is the peer reviewed version of: Khamis, M. M., Adamko, D. J. and El-Aneed, A. (2015), Mass Spec Rev., published in final form at https://doi.org/10.1002/mas.21455.

FIGURE 1. Strategies in metabolomic analysis for biomarker discovery.

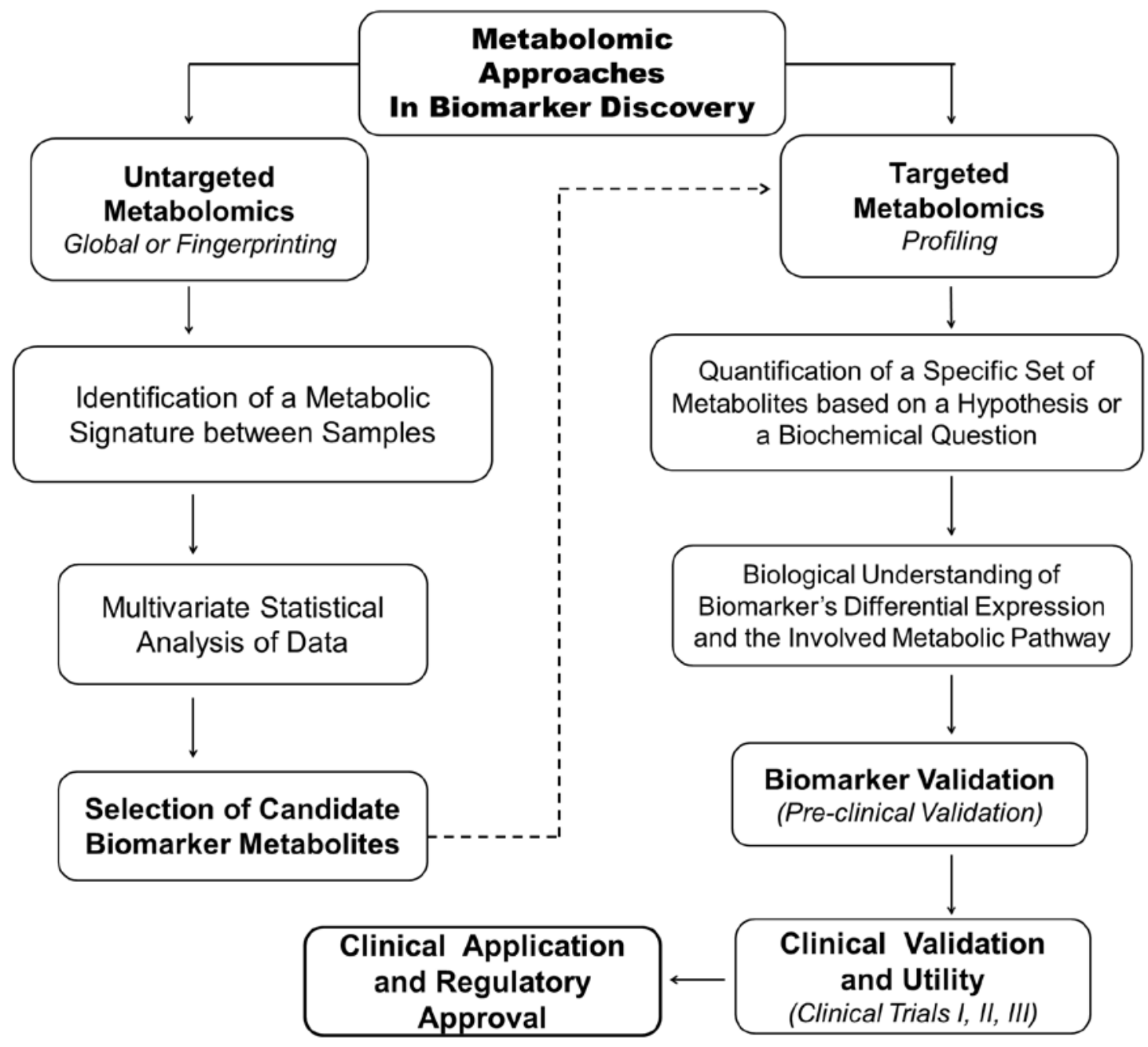


This is the peer reviewed version of: Khamis, M. M., Adamko, D. J. and El-Aneed, A. (2015), Mass Spec Rev., published in final form at https://doi.org/10.1002/mas.21455.

FIGURE 2. Base-peak chromatogram of the urinary profiles before and after the incorporation of LLE for the determination of carboxylic acid sub metabolome using pre-column derivatization followed by RPLC-FTICR-MS, adopted from ref (Peng \& Li, 2013), reprinted with permission.
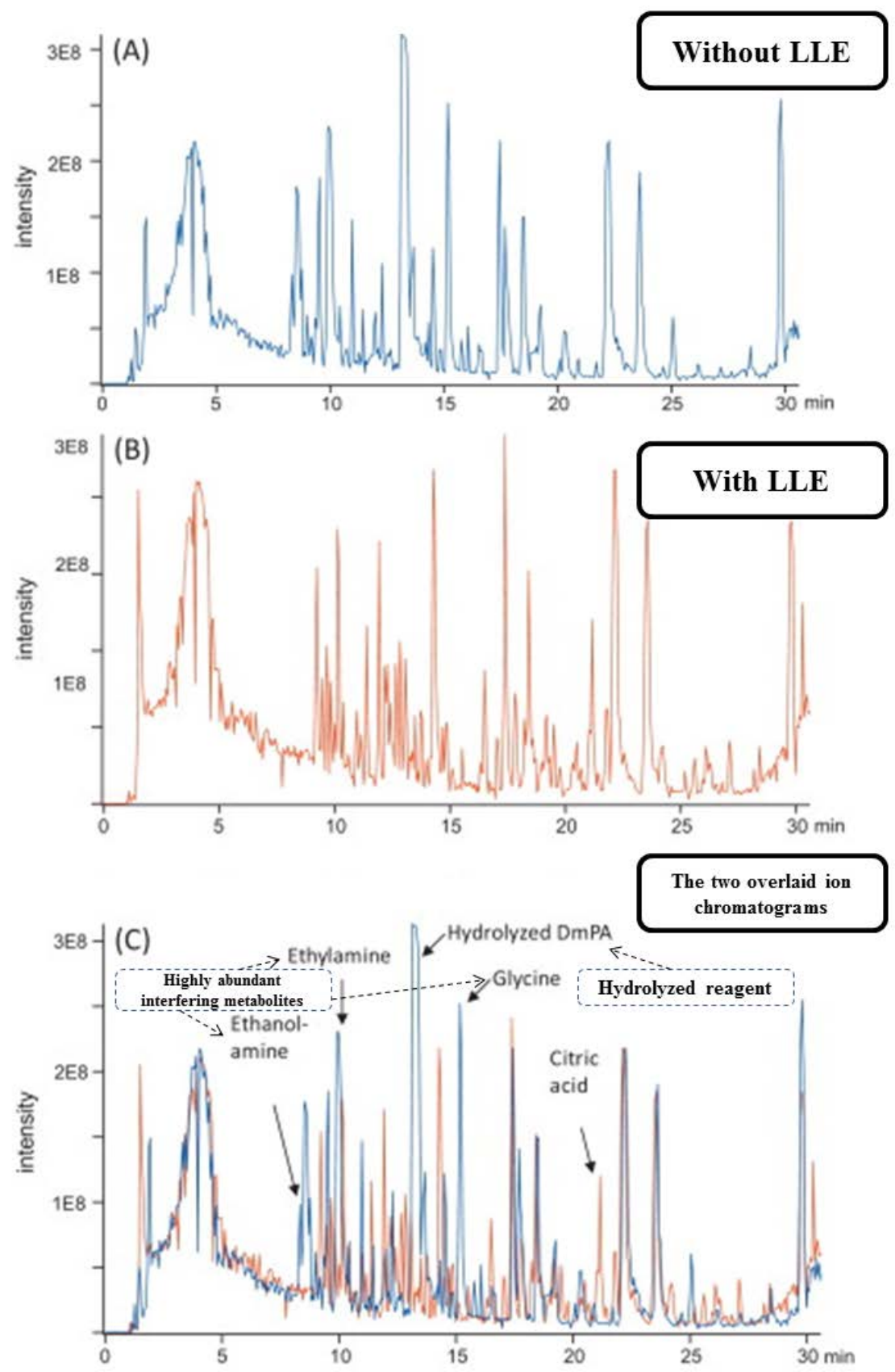
This is the peer reviewed version of: Khamis, M. M., Adamko, D. J. and El-Aneed, A. (2015), Mass Spec Rev., published in final form at https://doi.org/10.1002/mas.21455.

FIGURE 3. Online SPE extraction of (A) $10 \mu \mathrm{L}$ saline solution and (B) $10 \mu \mathrm{L}$ rat urine spiked with internal standards coupled to direct infusion analysis using ESI in positive mode and TOF-MS detection (LCT, Micromass). Internal standards used: 1. Indole-3-acetic acid-d2, 2. 11-aminoundecanoic acid, 3. Melatonin-d4, ref (Dettmer, Aronov \& Hammock, 2007), with permission.
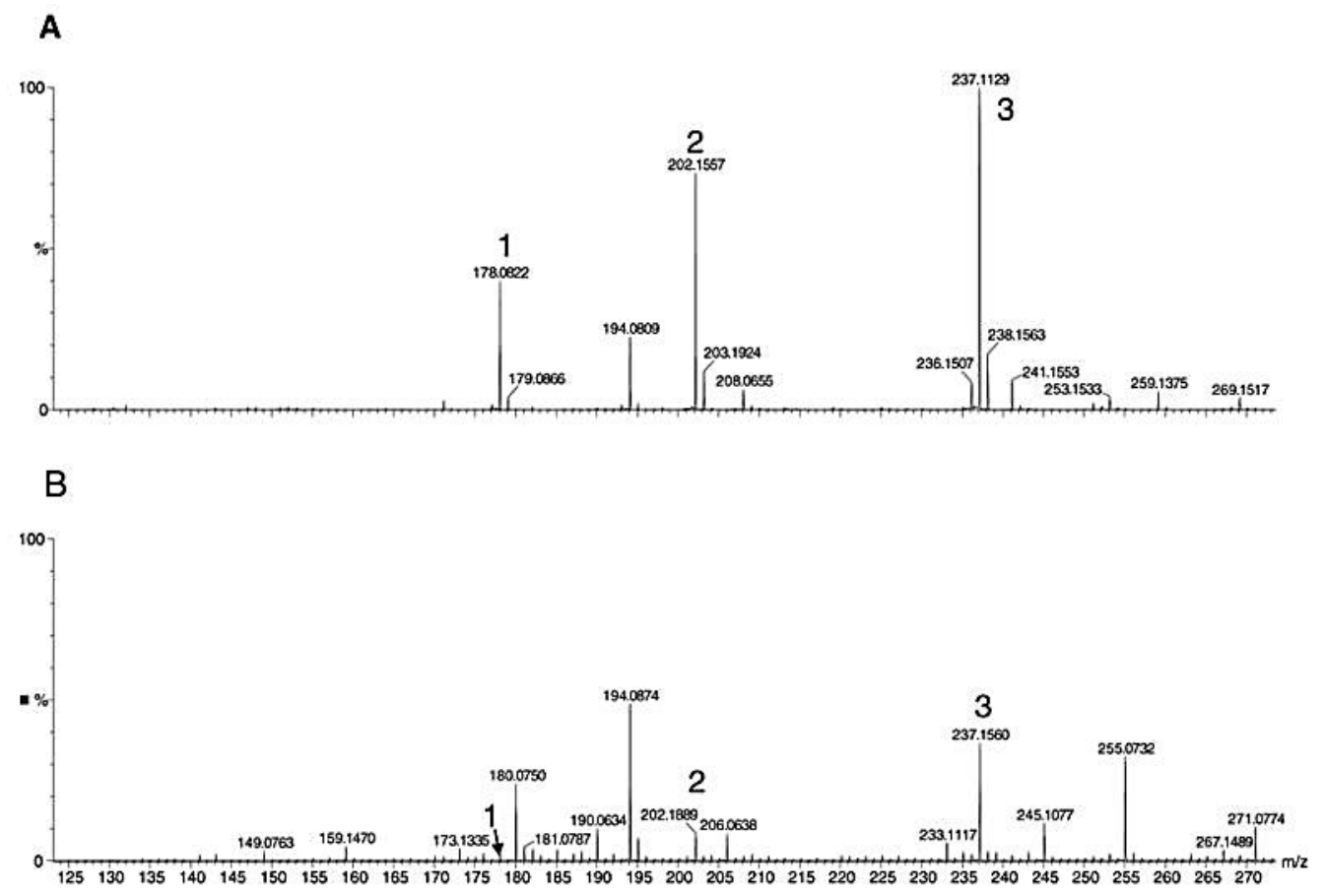
This is the peer reviewed version of: Khamis, M. M., Adamko, D. J. and El-Aneed, A. (2015), Mass Spec Rev., published in final form at https://doi.org/10.1002/mas.21455.

FIGURE 4. General scheme of the trimethylsilylation (BSTFA) and alkylation (ECF) reactions in GCMS.<smiles>[R]C(=O)O[Si](C)(C)C</smiles><smiles>[R]CO[Si](C)(C)C</smiles><smiles>[R]CN[Si](C)(C)C</smiles>

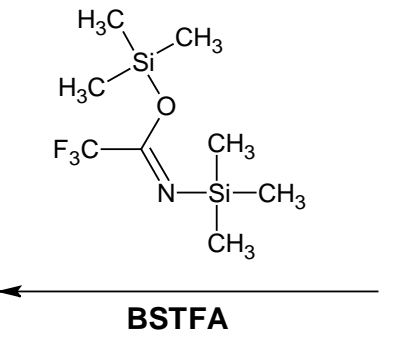

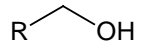

$$
\mathrm{R} \mathrm{NH}_{2}
$$<smiles>[R]C(=O)O</smiles><smiles>CCOC(=O)Cl</smiles>

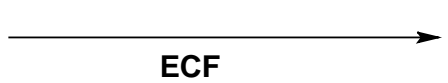<smiles>[R]C(=O)OCC</smiles><smiles>[R]COC(=O)OCC</smiles><smiles>[R]CNC(=O)OCC</smiles> 
This is the peer reviewed version of: Khamis, M. M., Adamko, D. J. and El-Aneed, A. (2015), Mass Spec Rev., published in final form at https://doi.org/10.1002/mas.21455.

FIGURE 5. Reaction scheme for profiling of carboxylic acid-containing metabolites using N-butyl-4aminomethylpyridinum iodide (BAMP) and N-hexyl-4-aminomethyl-pyridinum iodide (HAMP) reagents in rat urine.
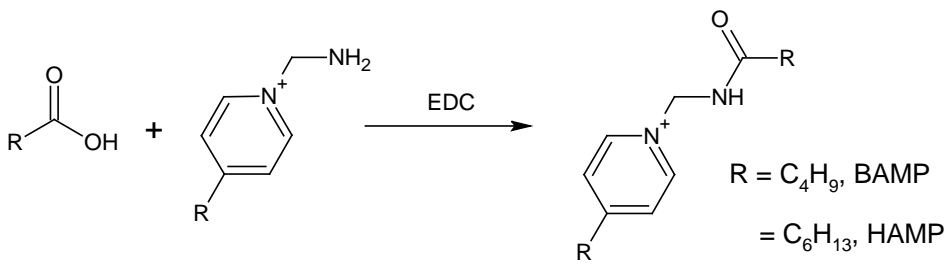
This is the peer reviewed version of: Khamis, M. M., Adamko, D. J. and El-Aneed, A. (2015), Mass Spec Rev., published in final form at https://doi.org/10.1002/mas.21455.

FIGURE 6. Chromatographic separation of white female AM mouse urine using (A) HPLC, (B) UPLC and Extracted ion chromatogram $\mathrm{m} / \mathrm{z}=401$ of white female AM mouse urine sample using either (C) HPLC, (D) UPLC, ref (Wilson et al., 2005), with permission

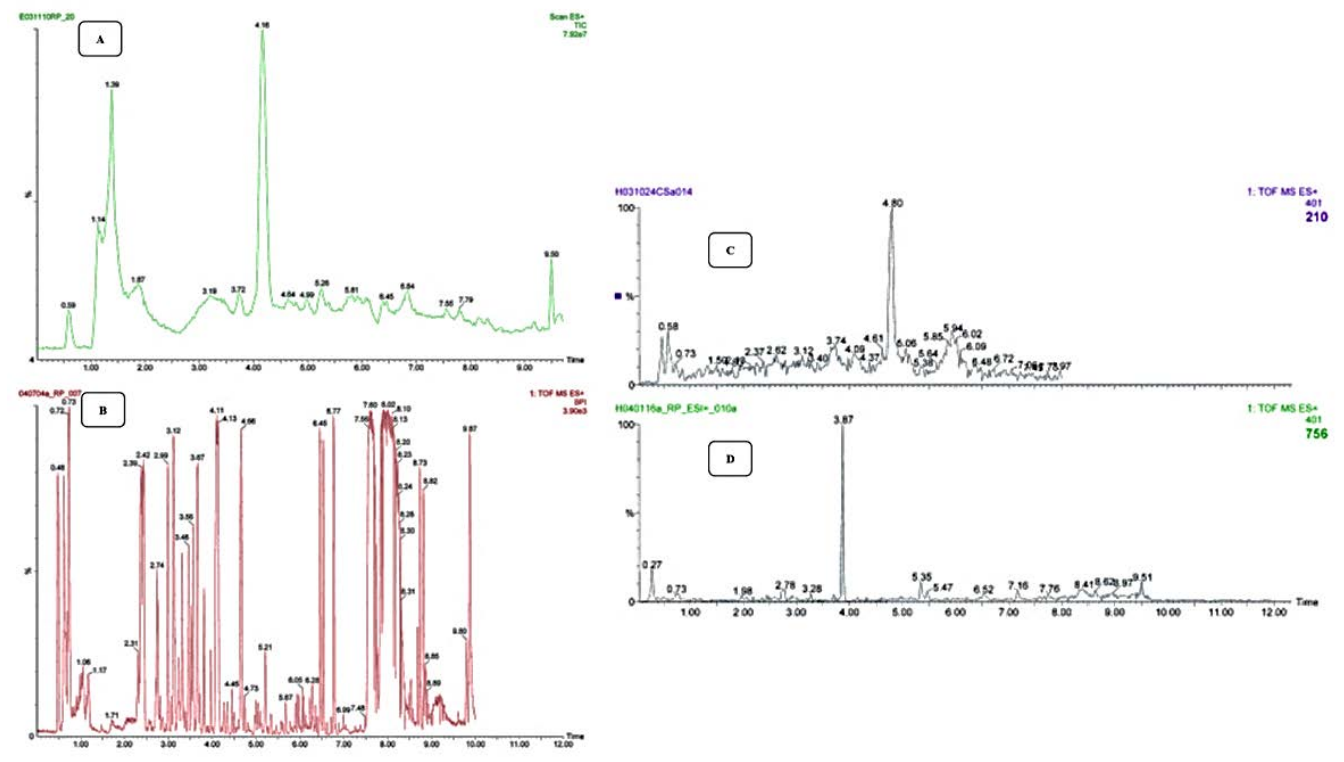


This is the peer reviewed version of: Khamis, M. M., Adamko, D. J. and El-Aneed, A. (2015), Mass Spec Rev., published in final form at https://doi.org/10.1002/mas.21455.

FIGURE 7. Schematic representation of light and heavy labeling for absolute and relative quantification of urinary metabolites in LC-MS based platform. Chromatogram adopted from ref (Guo \& Li, 2009) with permission.

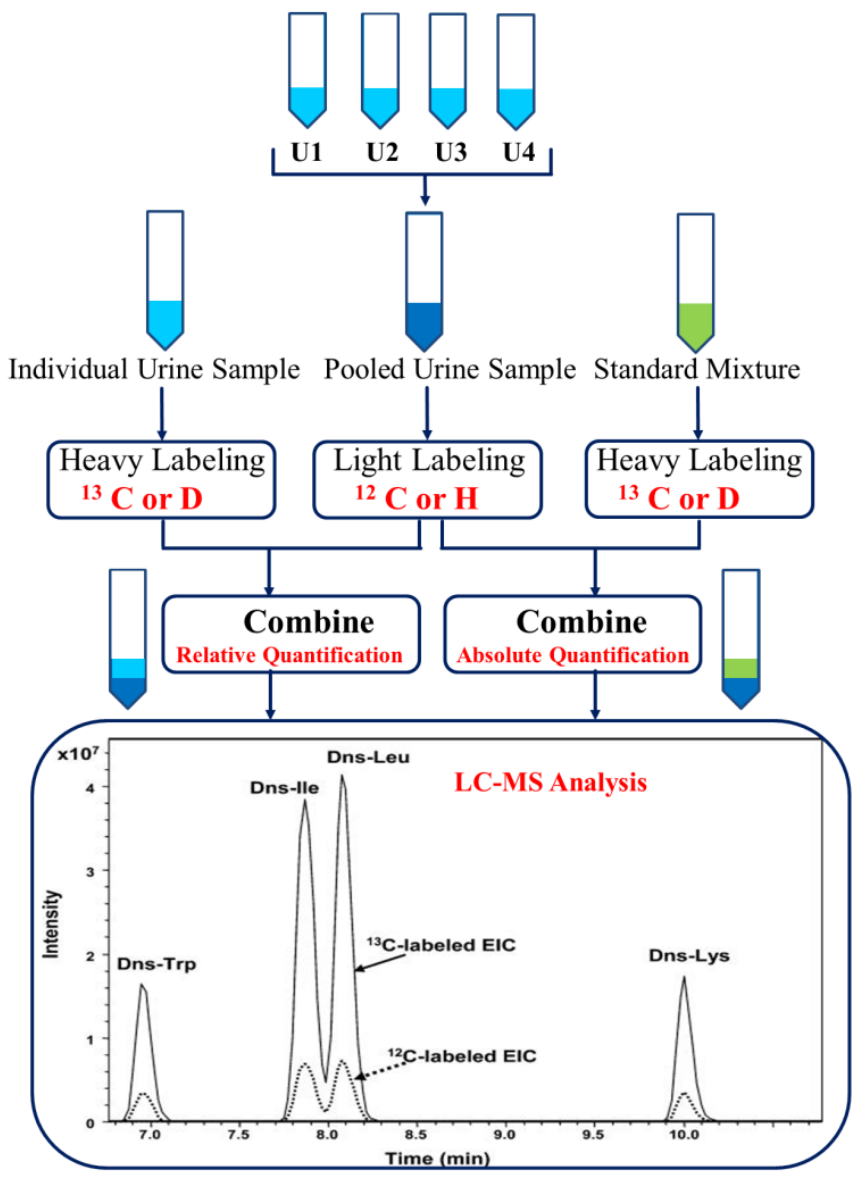


This is the peer reviewed version of: Khamis, M. M., Adamko, D. J. and El-Aneed, A. (2015), Mass Spec Rev., published in final form at https://doi.org/10.1002/mas.21455.

\section{REFERENCES}

Adamko D, Rowe BH, Marrie T, Sykes BD. 2007. Variation of metabolites in normal human urine. Metabolomics 3(4): 439-451.

Almstetter MF, Oefner PJ, Dettmer K. 2012. Comprehensive two-dimensional gas chromatography in metabolomics. Anal Bioanal Chem 402(6): 1993-2013.

Armitage EG, Barbas C. 2014. Metabolomics in cancer biomarker discovery: Current trends and future perspectives. J Pharm Biomed Anal 87: 1-11.

Bayer E. 2001. Recent advances in capillary electrophoresis/electrospray-mass spectrometry. Electrophoresis 22: 1251-1266.

Baños CE, Silva M. 2010. Liquid chromatography-tandem mass spectrometry for the determination of lowmolecular mass aldehydes in human urine. J Chromatogr B 878(7): 653-658.

Beger RD, Holland RD, Sun J, Schnackenberg LK, Moore PC, Dent CL, Devarajan P, Portilla D. 2008. Metabonomics of acute kidney injury in children after cardiac surgery. Pediatr Nephrol 23(6): 977-984.

Begley P, Francis-McIntyre S, Dunn WB, Broadhurst DI, Halsall A, Tseng A, Knowles J, Consortium H, Goodacre R, Kell DB. 2009. Development and Performance of a Gas Chromatography-Time-of-Flight Mass Spectrometry Analysis for Large-Scale Nontargeted Metabolomic Studies of Human Serum. Anal Chem 81(16): 7038-7046.

Bernini P, Bertini I, Luchinat C, Nincheri P, Staderini S, Turano P. 2011. Standard operating procedures for preanalytical handling of blood and urine for metabolomic studies and biobanks. J biomol NMR 49(3-4): 231-243.

Bicker W, Lämmerhofer M, Genser D, Kiss H, Lindner W. 2005. A case study of acute human chlorpyrifos poisoning: Novel aspects on metabolism and toxicokinetics derived from liquid chromatographytandem mass spectrometry analysis of urine samples. Toxicol lett 159(3): 235-251.

Boeniger MF, Lowry LK, Rosenberg J. 1993. Interpretation of urine results used to assess chemical exposure with emphasis on creatinine adjustments: a review. The American Industrial Hygiene Association Journal 54(10): 615-627.

Bondia-Pons I, Barri T, Hanhineva K, Juntunen K, Dragsted LO, Mykkänen H, Poutanen K. 2013. UPLC-QTOF/MS metabolic profiling unveils urinary changes in humans after a whole grain rye versus refined wheat bread intervention. Mol Nutr Food Res 57(3): 412-422.

Boomsma F, Alberts G, Van Eijk L, Schalekamp M. 1993. Optimal collection and storage conditions for catecholamine measurements in human plasma and urine. Clin chem 39(12): 2503-2508.

Borovecki F, Lovrecic L, Zhou J, Jeong H, Then F, Rosas H, Hersch S, Hogarth P, Bouzou B, Jensen R. 2005. Genome-wide expression profiling of human blood reveals biomarkers for Huntington's disease. Proc Natl Acad Sci U S A 102(31): 11023-11028.

Bouatra S, Aziat F, Mandal R, Guo AC, Wilson MR, Knox C, Bjorndahl TC, Krishnamurthy R, Saleem F, Liu P. 2013. The human urine metabolome. PloS one 8(9): e73076.

Boyland E, Wallace D, Williams D. 1955. The activity of the enzymes sulphatase and $\beta$-glucuronidase in the urine, serum and bladder tissue. Br J cancer 9(1): 62.

Buszewski B, Noga S. 2012. Hydrophilic interaction liquid chromatography (HILIC)-a powerful separation technique. Anal Bioanal Chem 402(1): 231-247. 
This is the peer reviewed version of: Khamis, M. M., Adamko, D. J. and El-Aneed, A. (2015), Mass Spec Rev., published in final form at https://doi.org/10.1002/mas.21455.

Chadha V, Garg U, Alon US. 2001. Measurement of urinary concentration: a critical appraisal of methodologies. Pediatr Nephrol 16(4): 374-382.

Chen J, Wang W, Lv S, Yin P, Zhao X, Lu X, Zhang F, Xu G. 2009. Metabonomics study of liver cancer based on ultra performance liquid chromatography coupled to mass spectrometry with HILIC and RPLC separations. Anal Chim Acta 650(1): 3-9.

Chen J-L, Huang Y-J, Pan C-H, Hu C-W, Chao M-R. 2011a. Determination of urinary malondialdehyde by isotope dilution LC-MS/MS with automated solid-phase extraction: a cautionary note on derivatization optimization. Free Radical Biol Med 51(9): 1823-1829.

Chen T, Xie G, Wang X, Fan J, Qiu Y, Zheng X, Qi X, Cao Y, Su M, Wang X. 2011b. Serum and urine metabolite profiling reveals potential biomarkers of human hepatocellular carcinoma. Mol Cell Proteomics 10(7): M110. 004945.

Cho S-H, Choi MH, Lee W-Y, Chung BC. 2009. Evaluation of urinary nucleosides in breast cancer patients before and after tumor removal. Clin biochem 42(6): 540-543.

Clarke DB, Lloyd AS, Botting NP, Oldfield MF, Needs PW, Wiseman H. 2002. Measurement of intact sulfate and glucuronide phytoestrogen conjugates in human urine using isotope dilution liquid chromatographytandem mass spectrometry with [13C3] isoflavone internal standards. Anal Biochem 309(1): 158-172.

Cone EJ, Caplan YH, Moser F, Robert T, Shelby MK, Black DL. 2009. Normalization of urinary drug concentrations with specific gravity and creatinine. J Anal Toxicol 33(1): 1-7.

Dai W, Huang Q, Yin P, Li J, Zhou J, Kong H, Zhao C, Lu X, Xu G. 2012. Comprehensive and Highly Sensitive Urinary Steroid Hormone Profiling Method Based on Stable Isotope-Labeling Liquid Chromatography-Mass Spectrometry. Anal Chem 84(23): 10245-10251.

Dettmer K, Aronov PA, Hammock BD. 2007. Mass spectrometry-based metabolomics. Mass Spectrom Rev 26(1): 51-78.

Dunn WB. 2008. Current trends and future requirements for the mass spectrometric investigation of microbial, mammalian and plant metabolomes. Phys biol 5(1): 011001.

Dunn WB, Broadhurst D, Ellis DI, Brown M, Halsall A, O'Hagan S, Spasic I, Tseng A, Kell DB. 2008. A GC-TOF-MS study of the stability of serum and urine metabolomes during the UK Biobank sample collection and preparation protocols. International journal of epidemiology, 37(suppl 1): i23-i30.

Dunn WB, Broadhurst DI, Atherton HJ, Goodacre R, Griffin JL. 2011. Systems level studies of mammalian metabolomes: the roles of mass spectrometry and nuclear magnetic resonance spectroscopy. Chem Soc Rev 40(1): 387-426.

Dunn WB, Ellis DI. 2005. Metabolomics: current analytical platforms and methodologies. TrAC, Trends Anal Chem 24(4): 285-294.

Dunn WB, Erban A, Weber RJ, Creek DJ, Brown M, Breitling R, Hankemeier T, Goodacre R, Neumann S, Kopka J. 2013. Mass appeal: metabolite identification in mass spectrometry-focused untargeted metabolomics. Metabolomics, 9(1): 44-66.

Eggink M, Wijtmans M, Ekkebus R, Lingeman H, de Esch IJP, Kool J, Niessen WM, Irth H. 2008. Development of a selective ESI-MS derivatization reagent: synthesis and optimization for the analysis of aldehydes in biological mixtures. Anal chem, 80(23): 9042-9051. 
This is the peer reviewed version of: Khamis, M. M., Adamko, D. J. and El-Aneed, A. (2015), Mass Spec Rev., published in final form at https://doi.org/10.1002/mas.21455.

Eggink M, Wijtmans M, Kretschmer A, Kool J, Lingeman H, de Esch IJP, Niessen WM, Irth H. 2010. Targeted LCMS derivatization for aldehydes and carboxylic acids with a new derivatization agent 4-APEBA. Anal Bioanal Chem 397(2): 665-675.

Fernández-Peralbo M, Luque de Castro M. 2012. Preparation of urine samples prior to targeted or untargeted metabolomics mass-spectrometry analysis. TrAC, Trends Anal Chem 41: 75-85.

Fiehn O, Kopka J, Dörmann P, Altmann T, Trethewey RN, Willmitzer L. 2000. Metabolite profiling for plant functional genomics. Nat biotechnol 18(11): 1157-1161.

Fiehn O, Robertson D, Griffin J, van der Werf M, Nikolau B, Morrison N, Sumner LW, Goodacre R, Hardy NW, Taylor C. 2007. The metabolomics standards initiative (MSI). Metabolomics 3(3): 175-178.

Gangl ET, Annan M, Spooner N, Vouros P. 2001. Reduction of signal suppression effects in ESI-MS using a nanosplitting device. Anal chem, 73(23): 5635-5644.

Gika HG, Theodoridis GA, Wilson ID. 2008a. Hydrophilic interaction and reversed-phase ultra-performance liquid chromatography TOF-MS for metabonomic analysis of Zucker rat urine. J Sep Sci 31(9): 1598-1608.

Gika HG, Theodoridis GA, Wilson ID. 2008b. Liquid chromatography and ultra-performance liquid chromatography-mass spectrometry fingerprinting of human urine: Sample stability under different handling and storage conditions for metabonomics studies. J Chromatogr A 1189(1): 314-322.

Gowda GN, Zhang S, Gu H, Asiago V, Shanaiah N, Raftery D. 2008. Metabolomics-based methods for early disease diagnostics. Expert Rev Mol Diagn 8(5): 617-633.

Griffiths WJ, Koal T, Wang Y, Kohl M, Enot DP, Deigner HP. 2010. Targeted metabolomics for biomarker discovery. Angew Chem Int Ed 49(32): 5426-5445.

Guo B, Chen B, Liu A, Zhu W, Yao S. 2012. Liquid chromatography-mass spectrometric multiple reaction monitoring-based strategies for expanding targeted profiling towards quantitative metabolomics. Curr Drug Metab, 13(9): 1226-1243.

Guo K, Ji C, Li L. 2007. Stable-isotope dimethylation labeling combined with LC-ESI MS for quantification of amine-containing metabolites in biological samples. Anal chem 79(22): 8631-8638.

Guo K, Li L. 2009. Differential 12C-/13C-isotope dansylation labeling and fast liquid chromatography/mass spectrometry for absolute and relative quantification of the metabolome. Anal chem 81(10): 3919-3932.

Guo, K., \& Li, L. 2010. High-performance isotope labeling for profiling carboxylic acid-containing metabolites in biofluids by mass spectrometry. Anal chem 82(21): 8789-8793.

Halket JM, Waterman D, Przyborowska AM, Patel RK, Fraser PD, Bramley PM. 2005. Chemical derivatization and mass spectral libraries in metabolic profiling by GC/MS and LC/MS/MS. J Exp Bot 56(410): 219-243.

Han J, Danell RM, Patel JR, Gumerov DR, Scarlett CO, Speir JP, Parker CE, Rusyn I, Zeisel S, Borchers CH. 2008. Towards high-throughput metabolomics using ultrahigh-field Fourier transform ion cyclotron resonance mass spectrometry. Metabolomics 4(2): 128-140.

Hasegawa M, Takenaka S, Kuwamura M, Yamate J, Tsuyama S. 2007. Urinary metabolic fingerprinting for amiodarone-induced phospholipidosis in rats using FT-ICR MS. Exp Toxicol Pathol 59(2): 115-120.

Hayama T, Sakaguchi Y, Yoshida H, Itoyama M, Todoroki K, Yamaguchi M, Nohta H. 2010. Fluorous derivatization combined with liquid chromatography/tandem mass spectrometry: a method for the selective and sensitive determination of sialic acids in biological samples. Rapid Commun Mass Spectrom 24(19): 2868-2874. 
This is the peer reviewed version of: Khamis, M. M., Adamko, D. J. and El-Aneed, A. (2015), Mass Spec Rev., published in final form at https://doi.org/10.1002/mas.21455.

Heavner DL, Morgan WT, Sears SB, Richardson JD, Byrd GD, Ogden MW. 2006. Effect of creatinine and specific gravity normalization techniques on xenobiotic biomarkers in smokers' spot and 24-h urines. J Pharm Biomed Anal 40(4): 928-942.

Hee SQ. 1993. Biological monitoring: an introduction. New York: John Wiley \& Sons. 141 p.

Hodson MP, Dear GJ, Griffin JL, Haselden JN. 2009. An approach for the development and selection of chromatographic methods for high-throughput metabolomic screening of urine by ultra pressure LC-ESIToF-MS. Metabolomics 5(2): 166-182.

Holmes E, Loo RL, Stamler J, Bictash M, Yap IK, Chan Q, Ebbels T, De lorio M, Brown IJ, Veselkov KA. 2008. Human metabolic phenotype diversity and its association with diet and blood pressure. Nature 453(7193): 396-400.

Hsu W-Y, Lin W-D, Tsai Y, Lin C-T, Wang H-C, Jeng L-B, Lee C-C, Lin Y-C, Lai C-C, Tsai F-J. 2011. Analysis of urinary nucleosides as potential tumor markers in human breast cancer by high performance liquid chromatography/electrospray ionization tandem mass spectrometry. Clin Chim Acta 412(19): 18611866.

Hu S, Wang J, Meijer J, leong S, Xie Y, Yu T, Zhou H, Henry S, Vissink A, Pijpe J. 2007. Salivary proteomic and genomic biomarkers for primary Sjögren's syndrome. Arthritis Rheum 56(11): 3588-3600.

Ito K, Kanada N, Inoue T, Furukawa K, Yamashita K, Tanaka N, Nakamura KT, Nishiya Y, Sogabe A, Yoshimoto T. 2002. Preliminary crystallographic studies of the creatinine amidohydrolase from Pseudomonas putida. Acta Crystallogr Sect D: Biol Crystallogr 58(12): 2180-2181.

Jacob P, Wilson M, Yu L, Mendelson J, Jones, RT. 2002. Determination of 4-hydroxy-3-methoxyphenylethylene glycol 4-sulfate in human urine using liquid chromatography-tandem mass spectrometry. Anal chem, 74(20): 5290-5296.

Jain K. 2010. The handbook of Biomarkers. New York: Springer. 10-11 p.

Jeng LB, Lo WY, Hsu WY, Lin WD, Lin CT, Lai CC, Tsai FJ. 2009. Analysis of urinary nucleosides as helper tumor markers in hepatocellular carcinoma diagnosis. Rapid Commun Mass Spectrom 23(11): 1543-1549.

Johnson CH, Gonzalez FJ. 2012. Challenges and opportunities of metabolomics. J Cell Physiol 227(8): 2975-2981.

Jung K, Pergande M. 1983. Particulate and free enzyme activity in urine as a result of the shedding of brushborder membranes from kidney. Clin Chim 29(2): 392-393.

Katajamaa M, Orešič M. 2007. Data processing for mass spectrometry-based metabolomics. J Chromatogr A 1158(1): 318-328.

Kim K, Taylor SL, Ganti S, Guo L, Osier MV, Weiss RH. 2011. Urine metabolomic analysis identifies potential biomarkers and pathogenic pathways in kidney cancer. OMICS 15(5): 293-303.

Kirwan J, Broadhurst D, Davidson R, Viant M. 2013. Characterising and correcting batch variation in an automated direct infusion mass spectrometry (DIMS) metabolomics workflow. Anal Bioanal Chem 405(15): 5147-5157.

Kirwan JA, Weber RJ, Broadhurst DI, Viant MR. 2014. Direct infusion mass spectrometry metabolomics dataset: a benchmark for data processing and quality control. Scientific Data 1.

Kretschmer A, Giera M, Wijtmans M, De Vries L, Lingeman H, Irth H, Niessen W. 2011. Derivatization of carboxylic acids with 4-APEBA for detection by positive-ion LC-ESI-MS (/MS) applied for the analysis of prostanoids and NSAID in urine. J Chromatogr B 879(17): 1393-1401. 
This is the peer reviewed version of: Khamis, M. M., Adamko, D. J. and El-Aneed, A. (2015), Mass Spec Rev., published in final form at https://doi.org/10.1002/mas.21455.

Kuehnbaum NL, Britz-McKibbin P. 2011. Comprehensive profiling of free and conjugated estrogens by capillary electrophoresis-time of flight/mass spectrometry. Anal chem 83(21): 8063-8068.

Kuklenyik Z, Ashley DL, Calafat AM. 2002. Quantitative detection of trichloroacetic acid in human urine using isotope dilution high-performance liquid chromatography-electrospray ionization tandem mass spectrometry. Anal chem 74(9): 2058-2063.

Kumar BS, Chung BC, Kwon OS, Jung BH. 2012. Discovery of common urinary biomarkers for hepatotoxicity induced by carbon tetrachloride, acetaminophen and methotrexate by mass spectrometry-based metabolomics. J Appl Toxicol 32(7): 505-520.

Kumar BS, Chung BC, Lee Y-J, Yi HJ, Lee B-H, Jung BH. 2011. Gas chromatography-mass spectrometry-based simultaneous quantitative analytical method for urinary oxysterols and bile acids in rats. Anal Biochem 408(2): 242-252.

Kumar BS, Lee Y-J, Yi HJ, Chung BC, Jung BH. 2010. Discovery of safety biomarkers for atorvastatin in rat urine using mass spectrometry based metabolomics combined with global and targeted approach. Anal Chim Acta 661(1): 47-59.

Lafaye A, Junot C, Gall RL, Fritsch P, Ezan E, Tabet JC. 2004. Profiling of sulfoconjugates in urine by using precursor ion and neutral loss scans in tandem mass spectrometry. Application to the investigation of heavy metal toxicity in rats. J Mass Spectrom 39(6): 655-664.

Lauridsen M, Hansen SH, Jaroszewski JW, Cornett C. 2007. Human urine as test material in $1 \mathrm{H}$ NMR-based metabonomics: recommendations for sample preparation and storage. Anal chem 79(3): 1181-1186.

Lei Z, Huhman DV, Sumner LW. 2011. Mass spectrometry strategies in metabolomics. J Biol Chem 286(29): 25435-25442.

Levine L, Fahy JP. 1945. Evaluation of urinary lead concentrations. I. The significance of the specific gravity. J Ind Hyg Toxicol 27: 217-223.

Lu W, Bennett BD, Rabinowitz JD. 2008. Analytical strategies for LC-MS-based targeted metabolomics. J Chromatogr B 871(2): 236-242.

Martínez-Lozano P, Rus J. 2010. Separation of Isomers< i $>$ L</i>-Alanine and Sarcosine in Urine by Electrospray Ionization and Tandem Differential Mobility Analysis-Mass Spectrometry. Journal of the American Society for Mass Spectrometry 21(7): 1129-1132.

Mikami T, Aoki M, Kimura T. 2012. The application of mass spectrometry to proteomics and metabolomics in biomarker discovery and drug development. Curr Mol Pharmacol 5(2): 301-316.

Miller RC, Brindle E, Holman DJ, Shofer J, Klein NA, Soules MR, O'Connor KA. 2004. Comparison of specific gravity and creatinine for normalizing urinary reproductive hormone concentrations. Clin chem 50(5): 924-932.

Monteiro M, Carvalho M, Bastos M, Guedes de Pinho P. 2013. Metabolomics analysis for biomarker discovery: advances and challenges. Curr Med Chem 20(2): 257-271.

Moon J-Y, Jung H-J, Moon MH, Chung BC, Choi MH. 2009. Heat-map visualization of gas chromatography-mass spectrometry based quantitative signatures on steroid metabolism. Journal of the American Society for Mass Spectrometry 20(9): 1626-1637.

Moore LE, Pfeiffer RM, Poscablo C, Real FX, Kogevinas M, Silverman D, García-Closas R, Chanock S, Tardón A, Serra C. 2008. Genomic DNA hypomethylation as a biomarker for bladder cancer susceptibility in the Spanish Bladder Cancer Study: a case-control study. The lancet oncology 9(4): 359-366. 
This is the peer reviewed version of: Khamis, M. M., Adamko, D. J. and El-Aneed, A. (2015), Mass Spec Rev., published in final form at https://doi.org/10.1002/mas.21455.

Nam H, Chung BC, Kim Y, Lee K, Lee D. 2009. Combining tissue transcriptomics and urine metabolomics for breast cancer biomarker identification. Bioinformatics 25(23): 3151-3157.

Pasikanti KK, Esuvaranathan K, Ho PC, Mahendran R, Kamaraj R, Wu QH, Chiong E, Chan ECY. 2010. Noninvasive urinary metabonomic diagnosis of human bladder cancer. J Proteome Res 9(6): 2988-2995.

Patti GJ, Yanes O, Siuzdak G. 2012. Innovation: Metabolomics: the apogee of the omics trilogy. Nat Rev Mol Cell Biol 13(4): 263-269.

Peng J, Li L. 2013. Liquid-liquid extraction combined with differential isotope dimethylaminophenacyl labeling for improved metabolomic profiling of organic acids. Anal Chim Acta 803: 97-105.

Pesek JJ, Matyska, MT, Fischer SM, Sana TR. 2008. Analysis of hydrophilic metabolites by high-performance liquid chromatography-mass spectrometry using a silica hydride-based stationary phase. J Chromatogr A 1204(1): 48-55.

Pesek JJ, Matyska MT, Loo JA, Fischer SM, Sana TR. 2009. Analysis of hydrophilic metabolites in physiological fluids by HPLC-MS using a silica hydride-based stationary phase. J Sep Sci 32(13): 2200-2208.

Pigini D, Cialdella A, Faranda P, Tranfo G. 2006. Comparison between external and internal standard calibration in the validation of an analytical method for 1-hydroxypyrene in human urine by high-performance liquid chromatography/tandem mass spectrometry. Rapid Commun Mass Spectrom 20(6): 1013-1018.

Pinto-Sietsma S-J, Janssen WM, Hillege HL, Navis G, de Zeeuw D, de Jong PE. 2000. Urinary albumin excretion is associated with renal functional abnormalities in a nondiabetic population. J Am Soc Nephrol 11(10): 1882-1888.

Plumb RS, Granger JH, Stumpf CL, Johnson KA, Smith BW, Gaulitz S, Wilson ID, Castro-Perez J. 2005. A rapid screening approach to metabonomics using UPLC and oa-TOF mass spectrometry: application to age, gender and diurnal variation in normal/Zucker obese rats and black, white and nude mice. Analyst 130(6): 844-849.

Plumb RS, Stumpf CL, Granger JH, Castro-Perez J, Haselden JN, Dear GJ. 2003. Use of liquid chromatography/time-of-flight mass spectrometry and multivariate statistical analysis shows promise for the detection of drug metabolites in biological fluids. Rapid Commun Mass Spectrom 17(23): 26322638.

Putluri N, Shojaie A, Vasu VT, Vareed SK, Nalluri S, Putluri V, Thangjam GS, Panzitt K, Tallman CT, Butler C. 2011. Metabolomic profiling reveals potential markers and bioprocesses altered in bladder cancer progression. Cancer research 71(24): 7376-7386.

Ramautar R, Mayboroda OA, Somsen GW, de Jong GJ. 2011. CE-MS for metabolomics: Developments and applications in the period 2008-2010. Electrophoresis 32(1): 52-65.

Ramautar R, Somsen GW, de Jong GJ. 2009. CE-MS in metabolomics. Electrophoresis 30(1): 276-291.

Ramautar R, Somsen GW, de Jong GJ. 2013. CE-MS for metabolomics: Developments and applications in the period 2010-2012. Electrophoresis 34(1): 86-98.

Remer T, Boye KR, Hartmann, MF, Wudy SA. 2005. Urinary markers of adrenarche: reference values in healthy subjects, aged 3-18 years. J Clin Endocrinol Metab 90(4): 2015-2021.

Rijk JC, Lommen A, Essers ML, Groot MJ, Van Hende JM, Doeswijk TG, Nielen MW. 2009. Metabolomics approach to anabolic steroid urine profiling of bovines treated with prohormones. Anal chem 81(16): 6879-6888.

University of Saskatchewan Author Manuscript, available April 16, 2017. 
This is the peer reviewed version of: Khamis, M. M., Adamko, D. J. and El-Aneed, A. (2015), Mass Spec Rev., published in final form at https://doi.org/10.1002/mas.21455.

Roberts LD, Souza AL, Gerszten RE, Clish CB. 2012. Targeted metabolomics. Current Protocols in Molecular Biology: 30.32. 31-30.32. 24.

Rocha SM, Caldeira M, Carrola J, Santos M, Cruz N, Duarte IF. 2012. Exploring the human urine metabolomic potentialities by comprehensive two-dimensional gas chromatography coupled to time of flight mass spectrometry. J Chromatogr A 1252: 155-163.

Roux A, Lison D, Junot C, Heilier J-F. 2011. Applications of liquid chromatography coupled to mass spectrometrybased metabolomics in clinical chemistry and toxicology: A review. Clin Biochem 44(1): 119-135.

Ryan D, Robards K, Prenzler P, Kendall M. 2011. Recent and potential developments in the analysis of urine: a review. Anal Chim Acta 684(1): 17-29.

Santa T. 2013. Derivatization in liquid chromatography for mass spectrometric detection. Drug Discoveries Ther $7(1)$.

Saude EJ, Obiefuna IP, Somorjai RL, Ajamian F, Skappak C, Ahmad T, Dolenko BK, Sykes BD, Moqbel R, Adamko DJ. 2009. Metabolomic biomarkers in a model of asthma exacerbation: urine nuclear magnetic resonance. Am J Respir Crit Care Med 179(1): 25-34.

Saude EJ, Skappak CD, Regush S, Cook K, Ben-Zvi, A, Becker A, Moqbel R, Sykes BD, Rowe BH, Adamko DJ. 2011. Metabolomic profiling of asthma: diagnostic utility of urine nuclear magnetic resonance spectroscopy. J Allergy Clin Immunol 127(3): 757-764. e756.

Scalbert A, Brennan L, Fiehn O, Hankemeier T, Kristal BS, van Ommen B, Pujos-Guillot E, Verheij E, Wishart D, Wopereis S. 2009. Mass-spectrometry-based metabolomics: limitations and recommendations for future progress with particular focus on nutrition research. Metabolomics 5(4): 435-458.

Schram KH. 1998. Urinary nucleosides. Mass Spectrom Rev 17(3): 131-251.

Seiwert B, Karst U. 2007. Simultaneous LC/MS/MS determination of thiols and disulfides in urine samples based on differential labeling with ferrocene-based maleimides. Anal chem 79(18): 7131-7138.

Shama N, Bai SW, Chung BC, Jung BH. 2008. Quantitative analysis of 17 amino acids in the connective tissue of patients with pelvic organ prolapse using capillary electrophoresis-tandem mass spectrometry. J Chromatogr B 865(1): 18-24.

Slupsky CM, Rankin KN, Wagner J, Fu H, Chang D, Weljie AM, Saude EJ, Lix B, Adamko, DJ, Shah S. 2007. Investigations of the effects of gender, diurnal variation, and age in human urinary metabolomic profiles. Anal chem 79(18): 6995-7004.

Soga T, Ohashi Y, Ueno Y, Naraoka H, Tomita M, Nishioka, T. 2003. Quantitative metabolome analysis using capillary electrophoresis mass spectrometry. J Proteome Res 2(5): 488-494.

Sreekumar A, Poisson LM, Rajendiran TM, Khan AP, Cao Q, Yu J, Laxman B, Mehra R, Lonigro RJ, Li Y. 2009. Metabolomic profiles delineate potential role for sarcosine in prostate cancer progression. Nature 457(7231): 910-914.

Stokvis E, Rosing H, López-Lázaro L, Schellens J, Beijnen J. 2004. Switching from an analogous to a stable isotopically labeled internal standard for the LC-MS/MS quantitation of the novel anticancer drug Kahalalide F significantly improves assay performance. Biomed Chromatogr 18(6): 400-402.

Stretch C, Eastman T, Mandal R, Eisner R, Wishart DS, Mourtzakis M, Prado CM, Damaraju S, Ball RO, Greiner R. 2012. Prediction of skeletal muscle and fat mass in patients with advanced cancer using a metabolomic approach. The Journal of nutrition 142(1): 14-21. 
This is the peer reviewed version of: Khamis, M. M., Adamko, D. J. and El-Aneed, A. (2015), Mass Spec Rev., published in final form at https://doi.org/10.1002/mas.21455.

Struck W, Siluk D, Yumba-Mpanga A, Markuszewski M, Kaliszan R, Markuszewski MJ. 2013. Liquid chromatography tandem mass spectrometry study of urinary nucleosides as potential cancer markers. J Chromatogr A 1283: 122-131.

Struys E, Jansen E, Gibson K, Jakobs C. 2005. Determination of the GABA analogue succinic semialdehyde in urine and cerebrospinal fluid by dinitrophenylhydrazine derivatization and liquid chromatographytandem mass spectrometry: application to SSADH deficiency. J Inherited Metab Dis 28(6): 913-920.

Sykes BD. 2007. Urine stability for metabolomic studies: effects of preparation and storage. Metabolomics 3(1): 19-27.

Theodoridis G, Gika HG, Wilson ID. 2011. Mass spectrometry-based holistic analytical approaches for metabolite profiling in systems biology studies. Mass Spectrom Rev 30(5): 884-906.

Theodoridis GA, Gika HG, Want EJ, Wilson ID. 2012. Liquid chromatography-mass spectrometry based global metabolite profiling: a review. Anal Chim Acta 711: 7-16.

Toyo'oka T. 2012. LC-MS determination of bioactive molecules based upon stable isotope-coded derivatization method. J Pharm Biomed Anal 69: 174-184.

Trivedi DK, lles RK. 2014a. Do not just do it, do it right: urinary metabolomics-establishing clinically relevant baselines. Biomed Chromatogr doi: 10.1002/bmc.3219.

Trivedi DK, lles RK. 2014b. HILIC-MS-based shotgun metabolomic profiling of maternal urine at 9-23 weeks of gestation-establishing the baseline changes in the maternal metabolome. Biomed Chromatogr doi: 10.1002/bmc.3266

Trivedi DK, Jones H, Shah A, Iles RK. 2012. Development of Zwitterionic Hydrophilic Liquid Chromatography (ZIC ${ }^{\circledR}$ HILIC-MS) Metabolomics Method for Shotgun Analysis of Human Urine. J Chromatogr Sep Tech 3(6): 144.

Turowski M, Yamakawa N, Meller J, Kimata K, Ikegami T, Hosoya K, Tanaka N, Thornton ER. 2003. Deuterium isotope effects on hydrophobic interactions: the importance of dispersion interactions in the hydrophobic phase. J Am Chem Soc 125(45): 13836-13849.

Tyburski JB, Patterson AD, Krausz KW, Slavík J, Fornace Jr AJ, Gonzalez FJ, Idle JR. 2009. Radiation metabolomics. 2. Dose-and time-dependent urinary excretion of deaminated purines and pyrimidines after sublethal gamma-radiation exposure in mice. Radiation research 172(1): 42-57.

Udert KM, Larsen TA, Biebow M, Gujer W. 2003. Urea hydrolysis and precipitation dynamics in a urine-collecting system. Water Research, 37(11): 2571-2582.

Urpi-Sarda M, Monagas M, Khan N, Llorach R, Lamuela-Raventós RM, Jáuregui O, Estruch R, Izquierdo-Pulido M, Andrés-Lacueva C. 2009. Targeted metabolic profiling of phenolics in urine and plasma after regular consumption of cocoa by liquid chromatography-tandem mass spectrometry. J Chromatogr A 1216(43): 7258-7267.

van de Merbel NC. 2008. Quantitative determination of endogenous compounds in biological samples using chromatographic techniques. TrAC, Trends Anal Chem 27(10): 924-933.

Van Doorn M, Vogels J, Tas A, Van Hoogdalem EJ, Burggraaf J, Cohen A, Van Der Greef J. 2007. Evaluation of metabolite profiles as biomarkers for the pharmacological effects of thiazolidinediones in Type 2 diabetes mellitus patients and healthy volunteers. Br J Clin Pharmacol 63(5): 562-574.

Van QN, Veenstra TD, Issaq HJ. 2011. Metabolic profiling for the detection of bladder cancer. Current urology reports 12(1): 34-40. 
This is the peer reviewed version of: Khamis, M. M., Adamko, D. J. and El-Aneed, A. (2015), Mass Spec Rev., published in final form at https://doi.org/10.1002/mas.21455.

Viau C, Lafontaine M, Payan J. 2004. Creatinine normalization in biological monitoring revisited: the case of 1hydroxypyrene. Int Arch Occup Environ Health 77(3): 177-185.

Villas-Bôas SG, Smart KF, Sivakumaran S, Lane GA. 2011. Alkylation or silylation for analysis of amino and nonamino organic acids by GC-MS? Metabolites 1(1): 3-20.

Vuckovic D. 2012. Current trends and challenges in sample preparation for global metabolomics using liquid chromatography-mass spectrometry. Anal Bioanal Chem 403(6): 1523-1548.

Wade D. 1999. Deuterium isotope effects on noncovalent interactions between molecules. Chem-Biol Interact 117(3): 191-217.

Wagner S, Scholz K, Donegan M, Burton L, Wingate J, Völkel W. 2006. Metabonomics and biomarker discovery: LC-MS metabolic profiling and constant neutral loss scanning combined with multivariate data analysis for mercapturic acid analysis. Anal chem 78(4): 1296-1305.

Wang X, Sun H, Zhang A, Wang P, Han Y. 2011. Ultra-performance liquid chromatography coupled to mass spectrometry as a sensitive and powerful technology for metabolomic studies. J Sep Sci 34(24): 34513459.

Wang X, Zhang A, Han Y, Wang P, Sun H, Song G, Dong T, Yuan Y, Yuan X, Zhang M. 2012. Urine metabolomics analysis for biomarker discovery and detection of jaundice syndrome in patients with liver disease. Mol Cell Proteomics 11(8): 370-380.

Want EJ, Wilson ID, Gika H, Theodoridis G, Plumb RS, Shockcor J, Holmes E, Nicholson, JK. 2010. Global metabolic profiling procedures for urine using UPLC-MS. Nat Protoc 5(6): 1005-1018.

Warrack BM, Hnatyshyn S, Ott K-H, Reily MD, Sanders M, Zhang H, Drexler DM. 2009. Normalization strategies for metabonomic analysis of urine samples. J Chromatogr B 877(5): 547-552.

Weimann A, Belling D, Poulsen HE. 2002. Quantification of 8-oxo-guanine and guanine as the nucleobase, nucleoside and deoxynucleoside forms in human urine by high-performance liquid chromatographyelectrospray tandem mass spectrometry. Nucleic Acids Res 30(2): e7-e7.

Williams DM, Sreedhar SS, Mickell JJ, Chan JC. 2002. Acute kidney failure: a pediatric experience over 20 years. Archives of pediatrics \& adolescent medicine 156(9): 893-900.

Wilson ID, Nicholson JK, Castro-Perez J, Granger JH, Johnson KA, Smith BW, Plumb RS. 2005. High resolution "ultra performance" liquid chromatography coupled to oa-TOF mass spectrometry as a tool for differential metabolic pathway profiling in functional genomic studies. J Proteome Res 4(2): 591-598.

Wishart DS, Jewison T, Guo AC, Wilson M, Knox C, Liu Y, Djoumbou Y, Mandal R, Aziat F, Dong E. 2013. HMDB 3.0-the human metabolome database in 2013. Nucleic Acids Res 41(D1): D801-D807.

Wittke S, Fliser D, Haubitz M, Bartel S, Krebs R, Hausadel F, Hillmann M, Golovko I, Koester P, Haller H. 2003. Determination of peptides and proteins in human urine with capillary electrophoresis-mass spectrometry, a suitable tool for the establishment of new diagnostic markers. J Chromatogr A 1013(1): 173-181.

Wojtowicz P, Zrostlíková J, Kovalczuk T, Schůrek J, Adam T. 2010. Evaluation of comprehensive two-dimensional gas chromatography coupled to time-of-flight mass spectrometry for the diagnosis of inherited metabolic disorders using an automated data processing strategy. J Chromatogr A 1217(51): 8054-8061.

Woo HM, Kim KM, Choi MH, Jung BH, Lee J, Kong G, Nam SJ, Kim S, Bai SW, Chung BC. 2009. Mass spectrometry based metabolomic approaches in urinary biomarker study of women's cancers. Clin Chim Acta 400(1): 63-69. 
This is the peer reviewed version of: Khamis, M. M., Adamko, D. J. and El-Aneed, A. (2015), Mass Spec Rev., published in final form at https://doi.org/10.1002/mas.21455.

Wu H, Xue R, Dong L, Liu T, Deng C, Zeng H, Shen X. 2009. Metabolomic profiling of human urine in hepatocellular carcinoma patients using gas chromatography/mass spectrometry. Anal Chim Acta 648(1): 98-104.

Wu Y, Li L. 2012. Determination of Total Concentration of Chemically Labeled Metabolites as a Means of Metabolome Sample Normalization and Sample Loading Optimization in Mass Spectrometry-Based Metabolomics. Anal chem 84(24): 10723-10731.

Xu X, Veenstra TD, Fox SD, Roman JM, Issaq HJ, Falk R, Saavedra JE, Keefer LK, Ziegler RG. 2005. Measuring fifteen endogenous estrogens simultaneously in human urine by high-performance liquid chromatography-mass spectrometry. Anal chem 77(20): 6646-6654.

Xu X, Ziegler RG, Waterhouse DJ, Saavedra JE, Keefer LK. 2002. Stable isotope dilution high-performance liquid chromatography-electrospray ionization mass spectrometry method for endogenous 2-and 4hydroxyestrones in human urine. J Chromatogr B 780(2): 315-330.

Yamashita K, Nakagawa R, Okuyama M, Honma S, Takahashi M, Numazawa M. 2008. Simultaneous determination of tetrahydrocortisol, allotetrahydrocortisol and tetrahydrocortisone in human urine by liquid chromatography-electrospray ionization tandem mass spectrometry. Steroids 73(7): 727-737.

Yang WC, Regnier FE, Adamec J. 2008. Comparative metabolite profiling of carboxylic acids in rat urine by CE-ESI MS/MS through positively pre-charged and 2H-coded derivatization. Electrophoresis 29(22): 4549-4560.

Zeisel SH, DaCosta KA, Fox JG. 1985. Endogenous formation of dimethylamine. Biochem J 232: 403-408.

Zhang A, Sun H, Wang P, Han Y, Wang X. 2012a. Modern analytical techniques in metabolomics analysis. Analyst 137(2): 293-300.

Zhang A, Sun H, Wu X, Wang X. 2012b. Urine metabolomics. Clin Chim Acta 414: 65-69.

Zhang T, Creek DJ, Barrett MP, Blackburn G, Watson DG. 2012c. Evaluation of coupling reversed phase, aqueous normal phase, and hydrophilic interaction liquid chromatography with Orbitrap mass spectrometry for metabolomic studies of human urine. Anal chem 84(4): 1994-2001.

Zhang Z, Bast RC, Yu Y, Li J, Sokoll LJ, Rai AJ, Rosenzweig JM, Cameron B, Wang YY, Meng X-Y. 2004. Three biomarkers identified from serum proteomic analysis for the detection of early stage ovarian cancer. Cancer research 64(16): 5882-5890.

Zheng J, Li L. 2012. Fragmentation of protonated dansyl-labeled amines for structural analysis of aminecontaining metabolites. International J Mass Spectrom 316: 292-299.

Zheng S, Yu M, Lu X, Huo T, Ge L, Yang J, Wu C, Li F. 2010. Urinary metabonomic study on biochemical changes in chronic unpredictable mild stress model of depression. Clin Chim Acta 411(3): 204-209.

Zhou R, Guo K, Li L. 2013. 5-Diethylamino-naphthalene-1-sulfonyl Chloride (DensCl): A novel triplex isotope labeling reagent for quantitative metabolome analysis by liquid chromatography mass spectrometry. Anal chem 85(23): 11532-11539.

Zhou R, Tseng C-L, Huan T, Li L. 2014. IsoMS: Automated Processing of LC-MS Data Generated by a Chemical Isotope Labeling Metabolomics Platform. Anal chem 86 (10): 4675-4679. 